
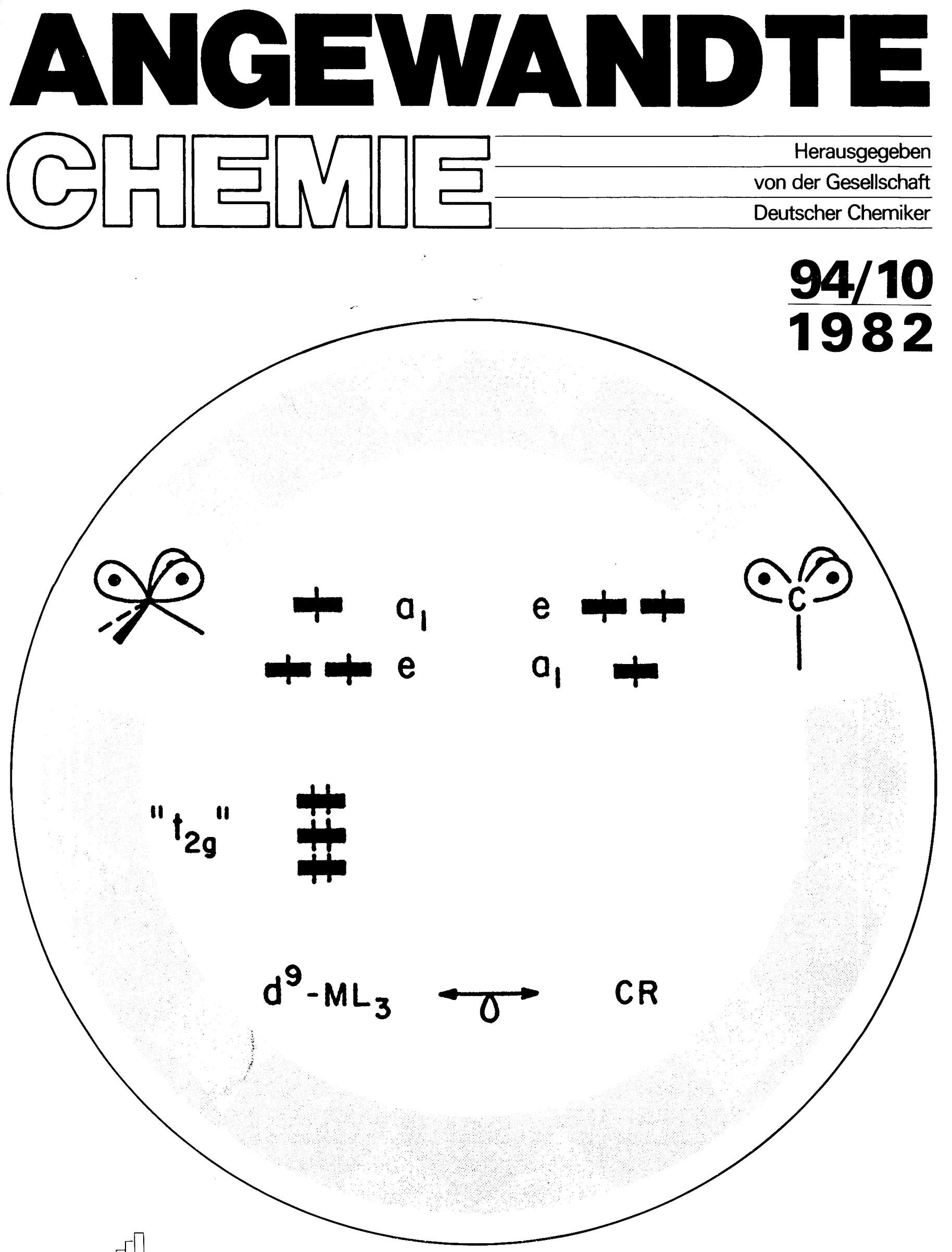


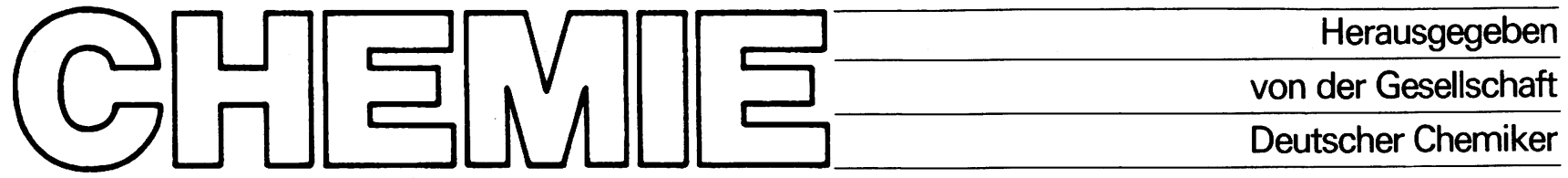

\section{R. Hoffmann}

725

H. Langhals

H. Mimoun

739

750

W. Bartmann, G. Beck
Brücken zwischen Anorganischer und Organischer Chemie (Nobel-Vortrag)

Polarität binärer Flüssigkeitsgemische

Sauerstoff-Übertragung von anorganischen und organischen Peroxiden auf organische Substrate: Ein gemeinsamer Mechanismus?

767 Prostacyclin und synthetische Analoga

\section{Inhalt - Zuschriften}

\section{B. Giese,}

S. Lachhein

J. Thiem, S. Köpper

M. Klessinger, J.-H. Cho

T. Sandow, J. Steidel, R. Steudel

A. Müller, W. Eltzner,

H. Bögge, R. Jostes

A. Müller, U. Reinsch-Vogell, E. Krickemeyer, H. Bögge

S. Dapperheld, E. Steckhan

R. Wienkamp, E. Steckhan

H. Irngartinger, A. Goldmann

J. Mulzer, A. Chucholowski

R. Hilgenfeld, W. Saenger

H.-J. Altenbach, B. Blech, J. A. Marco, E. Vogel

780

Addition von Alkyl-Radikalen an Alkine: Unterscheidung zwischen radikalischer und ionischer Nucleophilie

781 Synthese von Herzglycosiden: Evatromonosid und sein Anomer

782 Der Mehrwegemechanismus bei ${ }^{13} \mathrm{C}-{ }^{13} \mathrm{C}$-Fernkopplungskonstanten bicyclischer Kohlenwasserstoffe

782 Synthese von $S_{11}$ und $S_{13}$

783

-

784
$\left[\mathrm{Mo}_{4}{ }^{\mathrm{III}} \mathrm{S}_{4}(\mathrm{CN})_{12}\right]^{8-}$, ein Cluster mit hoher negativer Ladung und cubanartiger $\mathrm{Mo}_{4} \mathrm{~S}_{4}$-Einheit - Zur Bedeutung von Cyanothiomolybdaten für die präbiotische Evolution

Fixierung des Dithiosulfat-Ions $\mathrm{S}_{3} \mathrm{O}_{2}^{2-}$; Herstellung, Kristallstruktur und ESCA-Spektrum von $\left(\mathrm{PPh}_{4}\right)_{2}\left[\left(\mathrm{~S}_{2}\right) \mathrm{OMoS}_{2} \mathrm{MoO}\left(\mathrm{S}_{3} \mathrm{O}_{2}\right)\right]$

Oxidative Spaltung von Benzylestern mit Triarylamin-Radikalkationen selektive Deblockierung geschützter Carboxyfunktionen

Indirekte elektrochemische Regeneration von NADH mit einem Bipyridin-Rhodium(I)-Komplex als Elektronenüberträger

Elektronendichteverteilung in den Bindungen des verbrückten Bicyclo[1.1.0]butansystems 1,5-Dimethyltricyclo[2.1.0.0 $0^{2.5}$ pentan-3-on

Dreierdiastereoselektive Aldoladditionen von $\beta$-Lacton-enolaten an Aldehyde

Die Stetterschen Komplexe sind keine intramolekularen Einschlußverbindungen

7-Azanorbornadien 
G. Hilken, T. Kinkel, M. Schwamborn, J. Lex, H. Schmickler, E. Vogel

R. R. Schmidt, G. Grundler

G. Erker,

K. Engel, P. Vogel

J. Bücheler, N. Zeug, H. Kisch

L.-F. Tietze,

K.-H. Glüsenkamp, W. Holla

H. H. Paradies

R. Lapouyade, N. Hanafi, J.-P. Morand

P. Grenouillet, D. Neibecker, J. Poirier, I. Tkatchenko

H. Gotthardt, S. Schoy-Tribbensee, U. Feist

U. Schmidt,

A. Lieberknecht, U. Schanbacher, T. Beuttler, J. Wild

R. Herrmann, I. Ugi

T. Liese, G. Splettstößer, A. de Meijere

D. Spitzner, A. Engler, T. Liese, G. Splettstößer, A. de Meijere

H. Wamhoff, M. Ertas

B. von Maltzan

K. Bott

W. Sawodny, R. Grünes, H. Reitzle

A. G. Anastassiou, H. S. Kasmai, D. Hauger

J. Fuchs, A. Thiele, R. Palm

C. U. Pittman Jr., M. G. Richmond, M. Absi-Halabi, H. Beurich, F. Richter, H. Vahrenkamp
$790 \alpha$-Verknüpfte Disaccharide aus

$O$-( $\beta$-D-Glycopyranosyl)-trichloracetimidaten mit Trimethylsilyltrifluormethansulfonat als Katalysator

791 (2,9:6,12- $\eta$-2,3,5,6-Tetrakis(methylen)bicyclo[2.2.2]octan)zirconocen, eine isolierbare Bis(olefin)zirconium(II)-Verbindung

792 Zinksulfid als Katalysator der heterogenen Photoreduktion von Wasser

793 Ein einfacher und stereoselektiver Zugang zu den Secoiridoiden durch [4+2]-Cycloaddition von Alkyliden-1,3-dicarbonyl-Verbindungen

793 Bestimmung der Struktur von Dimethyldioctadecylammoniumchlorid-

- Vesikeln in Lösung durch Röntgen-Kleinwinkelstreuung; reversibler Phasenübergang

795 Photocyclisierung von 2-Aryl-1,1-difluorethylenen: Synthesen mono- und difluorierter polycyclischer Arene

Höhere Telomere bei der Pd-katalysierten Reaktion von Butadien mit Alkoholen

797 Stabile 1,3-Oxathiolylium-4-olate - Synthese und Cycloadditionen einer neuen Klasse mesoionischer Heteroarene

797 Einfache Herstellung von $\mathrm{N}$-Acyl-2-(diethoxyphosphoryl)-

- glycinestern und ihre Verwendung zum Aufbau von Dehydroaminosäureestern

798 Chirale zweikernige Carbenkomplexe aus $(R, R)-1-(1-$ Dimethylaminoethyl)-2-lithioferrocen und Hexacarbonylwolfram

799 Neue ringspannungsaktivierte Synthesebausteine:

- Allgemeiner Zugang zu 2-Chlor-2-cyclopropylidenacetaten

799 Reaktivität durch Ringspannung: Spirocyclopropylanellierte - tricyclische Gerüste durch Eintopf-Dreistufenreaktion

800 Selektive Sulfurierung und Photooxidation heteroanellierter 1,3-Oxazin-6-one zu 1,3-Thiazin-6-onen

$8012,3,7,8,12,13,17,18$-Octamethylporphyrinogen Synthese in praktisch quantitativer Ausbeute

802 Alkylierung von $\alpha$-Diazoketonen mit Alkyldiazonium-Ionen: Einfache Synthese von $\beta$-Alkoxyethylendiazoniumsalzen

803 Reversible $\mathrm{O}_{2}$-Anlagerung an polymere Schiffbase-Komplexe von - $\mathrm{V}^{\mathrm{II}}$ und $\mathrm{Mn}^{\mathrm{II}}$ sowie deren Verwendung als Oxidationskatalysatoren

803 Erzeugung und NMR-Nachweis der Anionen $N$-Methyl1-benzazocinid, 1-Benzazocindiid und Dibenz[b,g]azocindiid; eine Familie aromatischer $\pi$-Überschußverbindungen „ $\gamma-\mathrm{PW}_{12} \mathrm{O}_{40}^{3-c}$, ein Heteropolyanion mit Clathratcharakter

Photoinitiierte Hydrosilylierung in Gegenwart tetraedrischer Heterometallcluster: Katalyse durch intakte Cluster

Vollständiges Manuskript der so gekennzeichneten Zuschriften siehe Angew. Chem. Suppl. 1982, Nr. 10 
Konkordanz (September-Hefte 1982)

Die folgende Liste enthält die Namen aller Autoren von Aufsätzen und Zuschriften, die in den September-Heften der deutschen und englischen Ausgaben der ANGEWANDTEN CHEMIE veröffentlicht wurden. Die in eckigen Klammern stehenden und mit „IE“ bzw. „S“ gekennzeichneten Seitenzahlen beziehen sich auf die englische bzw. auf die SupplementAusgabe der Angewandten Chemie.

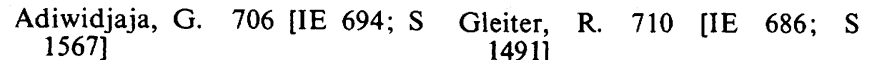
1567]

Basset, J. M. 722 [IE 687]

Benecke, I. 709 [IE 709; S 1605]

Ben Taarit, Y. 722 [IE 687]

Bermel, W. 703 [IE 689]

Berndt, A. 716 [IE 690]

Bittler, D. 718 [IE 696]

Boche, G. 703 [IE 688; S 1535]

Boland, W. 659 [IE 643]

Denis, J.-M. 715 [IE 690; S

1515]

Dogan, B. 721 [IE 708; S 1499]

Dower, W. V. 712 [IE 685; S 1545]

Ege, G. 715 [IE 698; S 1508]

Eiben, R. 703 [IE 688; S 1535]

Eierdanz, H. 716 [IE 690]

Elleuch, B. 722 [IE 687]

Euler, K. 706 [IE 693]

Förster, H. 703 [IE 689]

Francke, W. 704 [IE 698; S

1556]

Gilbert, K. 715 [IE 698; S 1508]
Goltz, M. 711 [IE 695]

Gubernator, K. 710 [IE 686; S 1491]

Guillemin, J.-C. 715 [IE 690; S 1515]

Hafner, K. 711, 721 [IE 695, 708; S - , 1499]

Heck, R. 715 [IE 698; S 1508]

Herrmann, W. A. 713 [IE 707; $S$ 1575]

Hofmann, P. 708 [IE 691]

Hofmeister, H. 718 [IE 696]

Jaenicke, L. 658 [IE 643]

Kaim, W. 712 [IE 700]

Kalinowski, H.-O. 706 [IE 693]

Kasack, V. 712 [IE 700]

Kervennal, J. 722 [IE 687]

Kessler, H. 7.03 [IE 689]

Klärner, F.-G. 721 [IE 708; S 1499]

König, W. A. 709 [IE 709; S 1605]

Kriechbaum, G. W. 713 [IE 707; S 1575]
Krziwanek, F. von 717 [IE 706]

Laurent, H. 718 [IE 696]

Mackenroth, W. 704 [IE 698; S 1556]

Maier, G. 706 [IE 693]

Marx, R. 717 [IE 706]

Müller, J. 708 [IE 691]

Nickisch, K. 718 [IE 696]

Nickolson, R. 718 [IE 696]

Nimmesgern, H. 706 [IE 694; S

1567]

Ott, W. 720 [IE 697; S 1479]

Panzer, B. 717 [IE 706]

Peters, K. 720 [IE 697; S 1479]

Petzoldt, K 718 [IE 696]

Pfisterer, H. 713 [IE 707; S 1575]

Prelog, V. 696 [IE 654]

Pusch, W. 670 [IE 660]

Range, K.-J. 717 [IE 706]

Rau, F. 717 [IE 706]

Ridder, H. 718 [IE 700; S

1525]

Roth, W. R. 721 [IE 708; S 1499]
Schaumann, E. 706 [IE 694; S 1567]

[IE 697; S 1479]

Seebach, D. 696 [IE 654]

Sellmann, D. 708 [IE 691]

Thiel, W. 703 [IE 688; S 1535]

Thometzek, P. 707 [IE 692]

Vollhardt, K. P. C. 712 [IE 685; S 1545]

Walch, A. 670 [IE 660]

Warwel, S. 718 [IE 700; S 1525]

Weiss, J. 719 [IE 705]

Werner, $\mathrm{H}, 707$ [IE 692]

Wiechert, R. 718 [IE 696]

Winkelmüller, W. 718 [IE 700 ; S 1525]

Zabel, R. 717 [IE 706]

Ziegler, M. L. 713 [IE 707; S 1575]

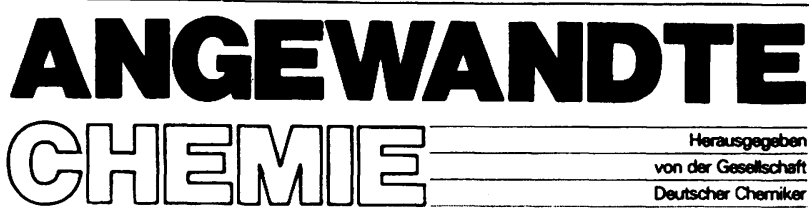

Kuratorium :

J. Thesing, K. H. Büchel, K. Decker, B. Franck, J.-M. Lehn,

H. Pommer, H. Schmidbaur, H. G. von Schnering,

G. Tölg, E. Vogel, A. Weiss, K. Weissermel

Redaktion:

O. Smrekar, P. Gölitz, G. Kruse

Pappelallee 3, D-6940 Weinheim

Telephon (06201) 602-1 Telex 465516 vchwh d

Verlag und Anzeigenabteilung:

Verlag Chemie GmbH

Postfach 1260/1280, D-6940 Weinheim

Telephon (06201) 602-1 Telex 465516 vchwh d

Erscheinungsweise: Monatlich.

Bestellungen richten Sie bitte an Ihre Fachbuchhandlung oder unmittelbar an den Verlag.

Adressenänderungen, Reklamationen: Bitte der Stelle mitteilen, die die Zeitschrift zustellt: dem örtlichen Zeitungsamt, der Sortimentsbuchhandlung oder dem Verlag.

Anzeigen: Zur Zeit gilt die Anzeigenpreisliste 22 vom 1. 10.1980 .
Buchbesprechungen: Die Redaktion behält sich bei der Besprechung von Büchern, die unverlangt zur Rezension eingehen, eine Auswahl vor. Nicht rezensierte Bücher werden nicht zurückgesandt.

Bezugspreis: Bezug durch den Verlag jährlich DM 298.- zuzüglich Versandgebühren. Einzelheft DM 30.-. In diesen Preisen sind 6,5\% Mehrwertsteuer enthalten.

Die Bezugsbedingungen für Mitglieder der Gesellschaft Deutscher Chemiker (GDCh) werden auf Anfrage von der Geschäftsstelle mitgeteilt. Geschäftsstelle der GDCh: Postfach 900440, D-6000 Frankfurt. Telephon (0611) 79171. Telex 412526 gmelin d für gdch. Postscheckkonto: 143671-600 Frankfurt.

Lieferung: Im Gebiet der Bundesrepublik Deutschland durch Postzeitungsvertrieb oder durch den Sortimentsbuchhandel, nach dem Ausland direkt unter Kreuzband oder ebenfalls durch den Sortimentsbuchhandel. Lieferung erfolgt auf Rechnung und Gefahr des Empfängers. Gerichtsstand und Erfüllungsort: Weinheim.

Abbestellungen: Bis spätestens 3 Monate vor Ablauf des Kalenderjahres.

For USA, Canada, Mexico, Central, Latin and South America: Published monthly by Verlag Chemie $\mathrm{GmbH}$, Weinheim, Federal Republic of Germany. For subscribers in the USA and Canada: $\$ 205.00$ including postage. Second-class postage paid at Jamaica, N. Y.-Printed in the Federal Republic of Germany.-Airfreighted and mailed in the United States by Publications Expediting Inc., 200 Meacham Avenue, Elmont, N.Y. 11003. Subscribers should place their orders through Verlag Chemie International Inc., Plaza Centre, Suite E, 1020 N.W. 6th Street, Deerfield Beach, Florida 33441 USA Postmasters: Send address changes for Angewandte Chemie to Verlag Chemie International Inc., Plaza Centre, Suite E, 1020 N.W. 6th Street, Deerfield Beach, Florida 33441. 


\title{
Polarität binärer Flüssigkeitsgemische
}

\author{
Von Heinz Langhals*
}

Im Gegensatz zu den schon gut untersuchten Polaritätseigenschaften von reinen Flüssigkeiten ist über die Polarität von Flüssigkeitsgemischen nur wenig bekannt, obwohl die Mehrzahl der mechanistischen sowie präparativen Studien chemischer Reaktionen nicht in reinen Phasen ausgeführt wird. Mit einer breit anwendbaren Zwei-Parameter-Gleichung läßt sich das polare Verhalten binärer Flüssigkeitsgemische als Funktion ihrer Zusammensetzung quantitativ beschreiben. Anhand dieser Gleichung kann man jetzt die bei Solvens-Mischungen beobachteten Abweichungen von der linearen Korrelation der Polaritätsskalen ebenso erklären wie die von Winstein bei der Solvolyse von tert-Butylchlorid gefundenen ungewöhnlichen Aktivierungsdaten. Anwendungen der Gleichung reichen von einem Schnelltest zur Bestimmung von Wasser in organischen Lösungsmitteln über die Untersuchung von Reaktionsmechanismen bis zur Polymerchemie.

\section{Einleitung}

Die Frage nach der Solvens-Polarität interessiert den Chemiker seit langem ${ }^{[1-4]}$. Nachdem sich makroskopische physikalische Größen wie die Dielektrizitätskonstante oder der Brechungsindex für die Klärung des chemischen Reaktionsverhaltens und der damit verbundenen molekularen Prozesse nur als begrenzt brauchbar erwiesen haben ${ }^{[1,5-8]}$, gelang mit der Entwicklung der empirischen Polaritätsskalen ein wesentlicher Fortschritt. Die älteste, von Winstein und Grunwald eingeführte $Y$-Skala ${ }^{[9-11]}$ nach Gleichung (1)

[*] Priv.-Doz. Dr. H. Langhals Chemisches Laboratorium der Universität Albertstraße 21, D-7800 Freiburg $\lg \frac{k}{k_{0}}=Y$

$k=$ Geschwindigkeitskonstante der Solvolyse von $\left(\mathrm{CH}_{3}\right)_{3} \mathrm{C}-\mathrm{Cl}$ im zu untersuchenden Medium

$k_{0}=$ Geschwindigkeitskonstante der Solvolyse von $\left(\mathrm{CH}_{3}\right)_{3} \mathrm{C}-\mathrm{Cl}$ in 80proz. Ethanol-Wasser

basiert auf der Solvolyse von tert-Butylchlorid und gibt vielfach den Einfluß des Lösungsmittels auf die Geschwindigkeit chemischer Reaktionen richtig wieder.

\section{Empirische Polaritätsskalen}

Die Winsteinschen $Y$-Werte werden heute allgemein als primäre Polaritätsskala verwendet. Von einer Solvolysere- 
aktion abgeleitet, ist die $Y$-Skala jedoch auf polare Medien begrenzt. Es wurde deshalb eine Reihe anderer Polaritätsskalen $^{[1]}$ auf der Basis von reaktionskinetischen oder spektroskopischen Daten entwickelt, die sich breiter anwenden lassen. Davon zeichnen sich die aus der Solvatochromie von Farbstoffen abgeleiteten Skalen durch einfache und präzise Meßbarkeit aus ${ }^{[12,13]}$. Eine Sonderstellung nimmt die von Dimroth und Reichardt $t^{[14,15]}$ entwickelte $E_{\mathrm{T}}(30)$ Skala ein, die sie zur am häufigsten verwendeten Skala werden ließ: Der als Meßsonde (Standardsubstrat) dienende solvatochrome Farbstoff Pentaphenylpyridiniophenolat 1

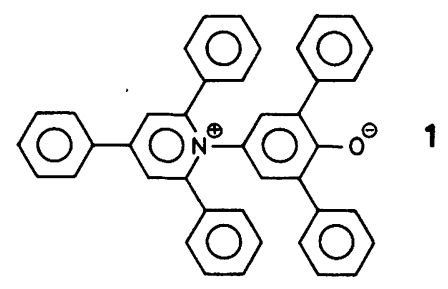

zeigt bei seinem längstwelligen UV/VIS-Absorptionsmaximum außerordentlich starke Verschiebungen der Bandenlage bei Änderung der Lösungsmittelpolarität und ist in fast allen üblichen Lösungsmitteln löslich. Die $E_{\mathrm{T}}(30)-$ Werte sind die Anregungsenergien $\left[\mathrm{kcal} \cdot \mathrm{mol}^{-1}\right]$ von 1 und werden aus $\lambda_{\max }$, der Solvatochromiebande von 1, nach Formel (2) berechnet.

$E_{\mathrm{T}}(30)=28590\left[\mathrm{kcal} \cdot \mathrm{nm} \cdot \mathrm{mol}^{-1}\right] / \lambda_{\max }$

Für viele Lösungsmittel besteht eine gute lineare Korrelation zwischen den $E_{\mathrm{T}}(30)$-Werten und den $Y$-Werten ${ }^{[1]}$. Diese lineare Korrelation gilt interessanterweise nicht nur für $E_{\mathrm{T}}(30)$ und $Y$, sondern für die meisten empirischen Polaritätsskalen ${ }^{[*]}$. Die Erkenntnis, daß es offenbar einen, wenn auch bisher nur empirisch faßbaren, für den molekularen Bereich zutreffenden Polaritätsbegriff gibt, war für die mechanistische Organische Chemie ebenso folgenreich wie für die präparative.

Einige wenige Polaritätsskalen korrelieren nicht mit der $E_{\mathrm{T}}(30)$-Skala (vgl. auch Abschnitt 3.2). Als Beispiel sei hier auf die Donizitätszahl $D N$ von Gutmann ${ }^{[16]}$ hingewiesen, die weniger die Polarität eines Lösungsmittels widerspiegelt als seine Lewis-Basizität. Die $D N$-Skala hat sich als brauchbar zur Beschreibung von Prozessen erwiesen, bei denen die Nucleophilie des Lösungsmittels eine wichtige Rolle spielt. In jüngster Zeit wurde versucht, solche weiteren Solvens-Eigenschaften zusammen mit der Polarität in einer Multi-Parameter-Gleichung zu erfassen ${ }^{[17,18]}$. Der Ansatz entspricht dem Prinzip der Polylinearität (PLL) von Palm $^{[19]}$ und ähnelt dem Multi-Parameter-Ansatz von Cra$\operatorname{mer}^{[20,21]}$.

\section{Binäre Gemische}

Die empirischen Polaritätsskalen bieten für viele praktische Anwendungen eine brauchbare Beschreibung des po-

[*] Ist ein Zusammenhang zwischen zwei Skalen nicht nur monoton, sondern sogar linear, so kann man daraus schließen, daß beide Skalen der gleichen Gesetzmäßigkeit folgen. laren Verhaltens von Flüssigkeiten. Erstaunlicherweise verschlechtert sich aber die für reine Lösungsmittel gültige lineare Korrelation der Polaritätsskalen wesentlich, wenn Lösungsmittelgemische mit einbezogen werden ${ }^{[1,15,22]}$. Gerade Lösungsmittelgemische sind aber für die Untersuchung von Solvens-Effekten besonders interessant, da sich durch Veränderung des Mischungsverhältnisses die Eigenschaften des einen Lösungsmittels kontinuierlich in die des anderen umwandeln lassen.

\subsection{Polarität als Funktion der Zusammensetzung}

Ein Schlüsselproblem war die quantitative Beschreibung der Polarität binärer Solvens-Gemische als Funktion ihrer Zusammensetzung mit einer nur wenige Parameter enthaltenden, breit anwendbaren Gleichung. Dimroth und Reichardt et al. ${ }^{[14,15]}$ untersuchten z. B. die Polarität der binären Gemische Methanol-Wasser, Ethanol-Wasser, AcetonWasser, Isopropylalkohol-Wasser, Pyridin-Wasser, 2,6-Lutidin-Wasser und Piperidin-Wasser als Funktion ihrer $\mathrm{Zu}$ sammensetzung unter Verwendung der $E_{\mathrm{T}}(30)$-Skala. Werden die $E_{\mathrm{T}}(30)$-Werte gegen die Zusammensetzung in Vol.$\%$ einer Komponente aufgetragen, so erhält man jedoch nicht wie zunächst erwartet werden mag eine lineare Beziehung, sondern stark gekrümmte Kurven, die wie auch in anderen Fällen ${ }^{[1,20,23]}$ nicht weiter interpretiert werden konnten. Eine Annäherung an den komplexen Funktionsverlauf wurde mit einer Taylor-Reihenentwicklung ${ }^{[24]}$ sowie mit mehreren Hyperbelfunktionen versucht ${ }^{[25]}$.

Vor kurzem wurde gefunden ${ }^{[26]}$, daß man beim Auftragen der $E_{\mathrm{T}}(30)$-Werte gegen den Logarithmus der molaren Konzentration an stärker polarer Komponente $\left(\ln c_{\mathrm{p}}\right) \mathrm{Kur}$ ven erhält, die einen linearen Abschnitt aufweisen. Figur la zeigt dies für das typische Gemisch $N$-tert-Butylformamid-Benzol.

Bei Solvolysereaktionen erhielt Tommila ${ }^{[27]}$ ebenfalls Kurven mit linearen Abschnitten, wenn bei binären Gemischen $\lg c_{\mathrm{p}}$ gegen die Freie Aktivierungsenthalpie der Solvolyse aufgetragen wurde. Der lineare Abschnitt wurde als Folge von nucleophiler Solvens-Beteiligung an der Reaktion interpretiert. Eine solche nucleophile Solvens-Beteiligung ist jedoch im Falle der $E_{\mathrm{T}}(30)$-Skala nicht möglich.

Mit der $E_{\mathrm{T}}(30)$-Skala, also mit Farbstoff 1 als Meßsonde, kann auch der Kurvenverlauf bei geringen Konzentrationen $c_{\mathrm{p}}$ untersucht werden. Die Kurve weicht in diesem Bereich von der Geraden ab und strebt für $c_{\mathrm{p}} \rightarrow 0$ gegen $E_{\mathrm{T}}^{0}(30)$, den $E_{\mathrm{T}}(30)$-Wert der reinen weniger polaren Komponente (vgl. Figur 1a). Mit der Zwei-Parameter-Gleichung (3) ist es nun gelungen, den gesamten Kurvenverlauf einschließlich des gekrümmten Bereichs in geschlossener Form zu erfassen ${ }^{[26]}$.

$E_{\mathrm{T}}(30)=E_{\mathrm{D}} \cdot \ln \left(\frac{c_{\mathrm{p}}}{c^{*}}+1\right)+E_{\mathrm{T}}^{0}(30)$

$E_{\mathrm{T}}(30)$ in Gleichung (3) ist die Polarität des binären Flüssigkeitsgemisches. $E_{\mathrm{T}}^{0}(30)$ und $c_{\mathrm{p}}$ haben die obengenannte Bedeutung. $E_{\mathrm{D}}$ und $c^{*}$ sind die Parameter der Gleichung; sie können graphisch ${ }^{[26,28]}$ oder mit dem Rechenprogramm POLAR $^{[26]}$ ermittelt werden. Die Gültigkeit von Gleichung (3) läßt sich durch Auftragen von $E_{\mathrm{T}}(30)$ gegen 
a)
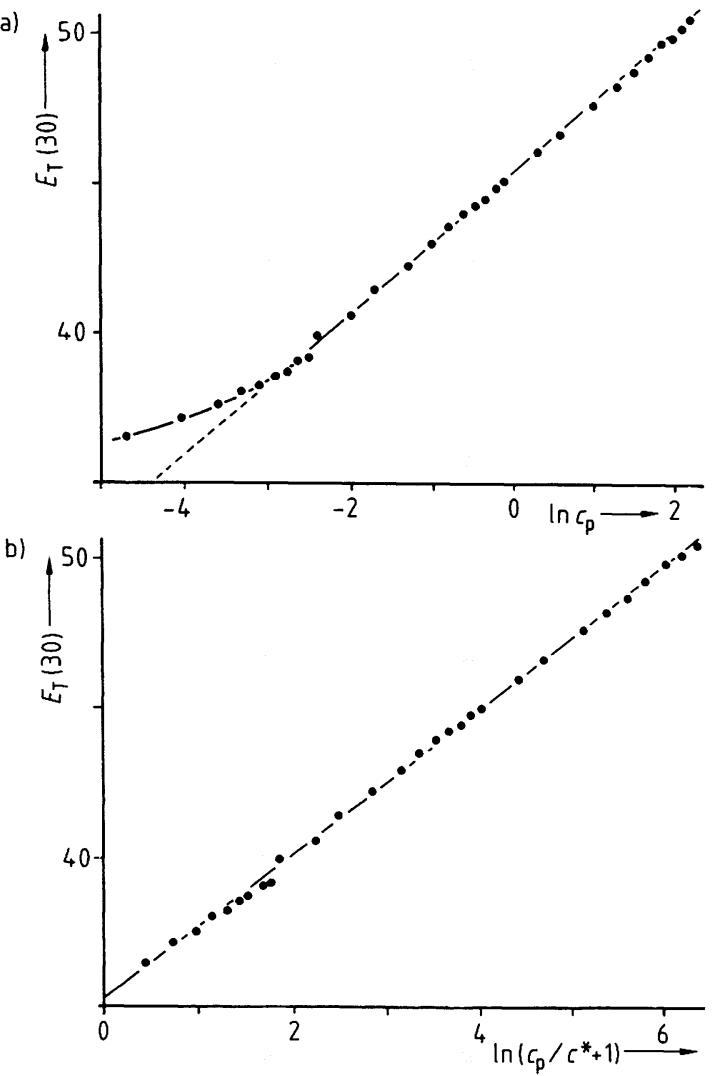

Fig. 1. $E_{\mathrm{T}}(30)$-Werte für das Gemisch $N$-tert-Butylformamid-Benzol a) als Funktion von $\left.\ln c_{\mathrm{p}}, \mathrm{b}\right)$ als Funktion von $\ln \left(c_{\mathrm{p}} / c^{*}+1\right)$ nach Gleichung (3).

$\ln \left(c_{\mathrm{p}} / c^{*}+1\right)$ zeigen. Hierbei resultiert eine Gerade, die in Figur $1 \mathrm{~b}$ für das Beispiel $\mathrm{N}$-tert-Butylformamid-Benzol über einen Bereich von drei Zehnerpotenzen für $c_{\mathrm{p}}$ dargestellt ist. Abweichungen von der Geraden sind statistisch und entsprechen den experimentellen Unsicherheiten.

\subsubsection{Die Parameter von Gleichung (3)}

Der Parameter $E_{\mathrm{D}}$ hat die Dimension einer Energie und ist ein $\mathrm{Maß}$ für die Empfindlichkeit, mit der die $E_{\mathrm{T}}(30)$ Skala auf eine relative Änderung von $c_{\mathrm{p}}$ anspricht. Der Parameter $c^{*}$ mit der Dimension einer Konzentration teilt die Kurve von Figur 1a in zwei Bereiche auf - eine Grenzwertbetrachtung von $c_{\mathrm{p}}$ verdeutlicht dies ${ }^{[26]}$. Für $c_{\mathrm{p}} \ll c^{*}$ ergibt sich, wie durch eine Reihenentwicklung von Gleichung (3) gezeigt werden kann, eine lineare Beziehung zwischen $E_{\mathrm{T}}(30)$ und $c_{\mathrm{p}}$. In diesem Konzentrationsbereich verhalten sich somit die Beiträge beider Komponenten zur Polarität des Gemisches additiv ${ }^{[*]}$. Für $c_{\mathrm{p}} \gg c^{*}$ wird dagegen eine lineare Beziehung zwischen $E_{\mathrm{T}}(30)$ und $\ln c_{\mathrm{p}}$ erhalten. Dieser Fall entspricht dem linearen Kurvenverlauf in Figur 1a. $c^{*}$ teilt also die Kurve in einen linearen und in einen logarithmischen Bereich und gibt an, ab welcher Schwelle die Wechselwirkung beider Flüssigkeiten nach Gleichung (3) in Erscheinung tritt. Der experimentell zugängliche Bereich von $c_{\mathrm{p}}$ erstreckt sich von der reinen weniger polaren Komponente, $c_{\mathrm{p}}=0$, bis zur reinen stärker polaren Komponente, $c_{\mathrm{p}}=c_{\mathrm{p}}^{\max }$. Ist nun $c^{*} \gg c_{\mathrm{p}}^{\max }$, so verhalten sich die

[*] Bei Auftragen der $E_{\mathrm{T}}(30)$-Werte gegen $\ln c_{\mathrm{p}}$ erhält man entsprechend eine gekrümmte Kurve: linker Ast in Figur 1 a.
Beiträge beider Komponenten zur Polarität des Gemisches, wie bereits erwähnt, über den gesamten Bereich annähernd additiv, und die mehrfach postulierte lineare Beziehung zwischen der Polarität und der Zusammensetzung des Gemisches ist erfüllt (Übersicht: ${ }^{[1,2]}$ ). Ist dagegen $c^{*}$ sehr klein, etwa $<0.06 \mathrm{~mol} \cdot \mathrm{L}^{-1}$, so ist der Zusammenhang zwischen der Polarität und $c_{\mathrm{p}}$ im experimentell relevanten Konzentrationsbereich nichtlinear.

\subsubsection{Weitere Polaritätsskalen}

Die bei der $E_{\mathrm{T}}(30)$-Skala gefundene Gesetzmäßigkeit nach Gleichung (3) wird auch bei anderen Polaritätsskalen festgestellt ${ }^{[29-31]}$ und deshalb in Gleichung (4) allgemein formuliert.

$P_{\mathrm{G}}=E_{\mathrm{D}} \cdot \ln \left(\frac{c_{\mathrm{p}}}{c^{*}}+1\right)+P_{\mathrm{G}}^{0}$

$P_{\mathrm{G}}$ in Gleichung (4) ist die Polarität des binären Flüssigkeitsgemisches nach einer empirischen Polaritätsskala und $P_{\mathrm{G}}^{0}$ der $P_{\mathrm{G}}$-Wert der reinen weniger polaren Komponente. $c_{\mathrm{p}}, c^{*}$ und $E_{\mathrm{D}}$ haben die gleiche Bedeutung wie in Gleichung (3). Beide Gleichungen zeigen naturgemäß gleiches Grenzverhalten (vgl. Abschnitt 3.1.1).

Die empirischen Polaritätsskalen, von denen eine Auswahl in Tabelle 1 aufgeführt ist, basieren auf unterschiedlichen Effekten, die zum Beispiel von der $\pi \rightarrow \pi^{*}$-Absorption (Nr. 1, 4-7), Charge-Transfer-Absorption (Nr. 3), Fluoreszenz $(\mathrm{Nr} .8,9)$ bis zur Solvolysereaktion (Nr. 2) reichen. Die $E_{\mathrm{T}}$ (1)-Skala (Nr. 5) gibt nicht nur die Polarität des Mediums wieder, sondern darüber hinaus auch noch seinen Wasserstoffbrücken-Donorcharakter.

Gleichung (4) ist eine nach allen Kriterien der untersuchten Polaritätsskalen gültige Beschreibung für das polare Verhalten binärer Flüssigkeitsgemische als Funktion ihrer Zusammensetzung ${ }^{[29]}$. Für einige Polaritätsskalen von Tabelle 1 ist der nach Gleichung (4) lineare Zusammenhang zwischen $P_{\mathrm{G}}$ oder $P_{\mathrm{G}}-P_{\mathrm{G}}^{0}$ und $\ln \left(c_{\mathrm{p}} / c^{*}+1\right)$ in Figur 2 dargestellt.

Einen Eindruck der Gültigkeitsbreite von Gleichung (4) vermittelt Tabelle 2, in der ihre Anwendung bei den neun

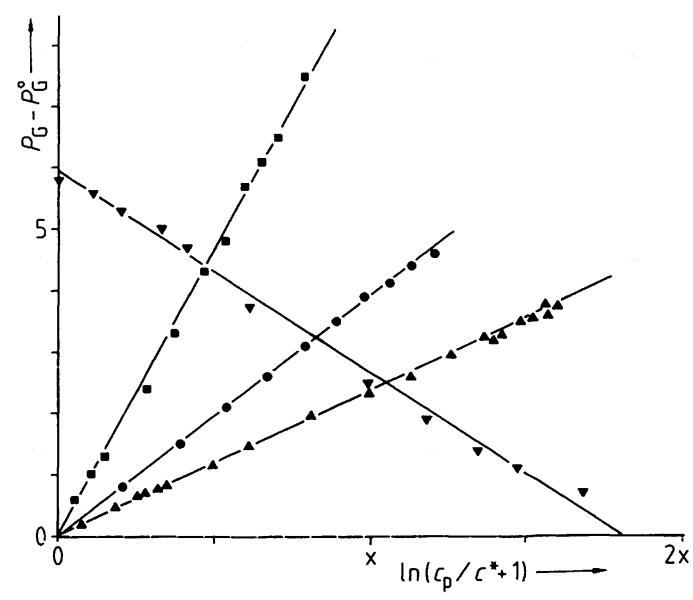

Fig. 2. Linearer Zusammenhang zwischen $P_{\mathrm{G}}$ und $\ln \left(c_{\mathrm{p}} / c^{*}+1\right)$ für verschiedene Polaritätsskalen (Gleichung (4)): $\Delta E_{\mathrm{T}}(30)$ Ethanol-1-Decanol, $\mathrm{x}=1$; - $Y$ Wasser-Methanol, $\mathrm{x}=1 ; \square Z$ Methanol-Aceton, $\mathrm{x}=2 ; \nabla \pi \mathrm{i}$ Ethanol$n$-Heptan, $\mathrm{x}=1$, Ordinate $P_{\mathrm{G}}-P_{\mathrm{G}}^{0}+5.8$. 
<smiles>CC(C)(C)Cl</smiles>

2

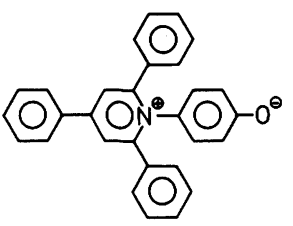

5

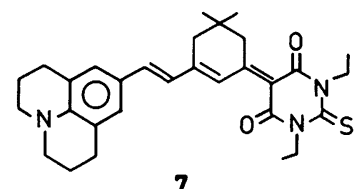

7

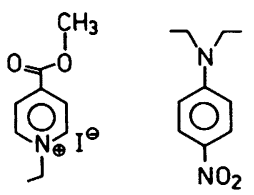

4

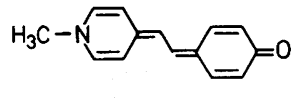

6

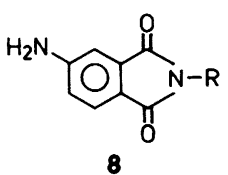

Polaritätsskalen von Tabelle 1 gezeigt wird. Aus der Anzahl und Verschiedenartigkeit der verwendeten Komponenten - es wurden polar protonische, dipolar aprotonische und unpolare Lösungsmittel, auch Aromaten miteinander kombiniert - ist zu folgern, daß Gleichung (4) eine übergreifende Beschreibung der Polarität von binären Gemischen ermöglicht, die nicht auf einzelne Lösungsmittelklassen $^{[38]}$ beschränkt ist. Besonders zu beachten ist ihre Gültigkeit bei Skala 5 und dem Gemisch Ethanol-Wasser $^{[29]}$, das stark zur H-Brückenbildung neigt (Tabelle 2, Nr. 54). Polaritätseffekte, die auf Wasserstoffbrücken zurückzuführen sind, werden somit ebenfalls von Gleichung (4) erfaßt.

\subsubsection{Sonderfälle}

Besonderheiten werden beim Lösungsmittelgemisch Wasser-1,4-Dioxan beobachtet: Bis zu einem Wassergehalt von ca. $50 \%$ wird bei Untersuchung mit der $E_{\mathrm{T}}(30)$ Skala normales Verhalten nach Gleichung (3) festgestellt.

Tabelle 1. Empirische Solvens-Polaritätsskalen.

\begin{tabular}{|c|c|c|c|c|c|}
\hline Nr. & Skala [a] & Meßsonde & Lösungsmittelabhängiger Prozeß & Definition $[b]$ & Lit. \\
\hline 1 & $E_{\mathrm{T}}(30)$ & 1 & $\pi \rightarrow \pi^{*}$-Absorption & $E_{\mathrm{T}}(30)=28590 / \lambda_{\max }[\mathrm{c}]$ & {$[14,15]$} \\
\hline 2 & $Y$ & 2 & Solvolyse relativ zur Solvolyse in $80 \% \mathrm{EtOH} / \mathrm{H}_{2} \mathrm{O}\left(k_{0}\right)$ & $Y=\lg \frac{k}{k_{0}}[\mathrm{~d}]$ & [32] \\
\hline 3 & $Z$ & 3 & CT-Absorption & $Z=28590 / \lambda_{\max }[c]$ & [12] \\
\hline 4 & $\pi_{i}^{*}$ & 4 & $\pi \rightarrow \pi^{*}$-Absorption & $\pi_{i}^{*}=28590 / \lambda_{\max }[\mathrm{c}, \mathrm{e}]$ & {$[17,18]$} \\
\hline 5 & $E_{\mathrm{T}}(1)$ & 5 & $\pi \rightarrow \pi^{*}$-Absorption & $E_{\mathrm{T}}(1)=28950 / \lambda_{\max }[\mathrm{c}]$ & [14] \\
\hline 6 & $M O E D$ & 6 & $\pi \rightarrow \pi^{*}$-Absorption & $M O E D=28590 / \lambda_{\max }[c]$ & {$[13,33,34]$} \\
\hline 7 & $\chi_{R}$ & 7 & $\pi \rightarrow \pi^{*}$-Absorption & $\chi_{\mathrm{R}}=28590 / \lambda_{\max }[\mathrm{c}]$ & [13] \\
\hline 8 & $S_{1}$ & $8, \mathrm{R}=\mathrm{CH}_{3}$ & Fluoreszenz & $S_{1}=28590 / \lambda_{\max }[\mathrm{c}]$ & {$[35-37]$} \\
\hline 9 & $S_{2}$ & $8, R=H$ & Fluoreszenz & $S_{2}=28590 / \lambda_{\max }[c]$ & [35] \\
\hline
\end{tabular}

[a] Andere Polaritätsskalen siehe [1]. [b] Bei $25^{\circ} \mathrm{C}$. [c] Molare Anregungs- bzw. Emissionsenergien in kcal $\cdot \mathrm{mol}^{-1}$; zur Umrechnung in SI-Einheiten ist mit dem Faktor 4.2 zu multiplizieren. [d] In $Y$-Einheiten; zur Umrechnung in $\mathrm{kcal} \cdot \mathrm{mol}^{-1}$ ist mit dem Faktor 0.733 zu multiplizieren. [e] In [17] sind die molaren Anregungsenergien in $\mathrm{cm}^{-1}$ angegeben; sie wurden für einen direkten Vergleich mit den anderen Skalen in $\mathrm{kcal} \cdot \mathrm{mol}^{-1} \mathrm{umgerechnet}^{-}$

Tabelle 2. Die Parameter $E_{\mathrm{D}}$ und $c^{*}$ von Gleichung (4) für binäre Solvens-Gemische.

\begin{tabular}{|c|c|c|c|c|c|c|c|c|c|c|}
\hline $\mathrm{Nr}$. & Komponenten [a] & $\begin{array}{l}c_{\mathrm{p}} \\
{[\mathrm{b}, \mathrm{c}]}\end{array}$ & $\begin{array}{l}\text { Meß- } \\
\text { sonde }\end{array}$ & $\begin{array}{l}P_{\mathrm{G}}^{0} \\
{[\mathrm{~d}, \mathrm{e}]}\end{array}$ & $\begin{array}{l}c^{*} \\
{[c, f]}\end{array}$ & $\begin{array}{l}E_{\mathrm{D}} \\
{[\mathrm{d}, \mathrm{f}]}\end{array}$ & $\begin{array}{l}\sigma\left(E_{\mathrm{D}}\right) \\
{[\mathrm{g}]}\end{array}$ & $\begin{array}{l}r \\
{[h]}\end{array}$ & $\begin{array}{l}\mathrm{n} \\
{[\mathrm{i}]}\end{array}$ & $\begin{array}{l}c_{\mathrm{k}} \\
{[\mathrm{c}, \mathrm{f}]}\end{array}$ \\
\hline 1 & 1-Butanol-Aceton & $0.01-10.9$ & 1 & 42.2 & 0.14 & 1.99 & 0.01 & 0.99952 & 31 & - \\
\hline 2 & Ethanol-Aceton & $0.02-17.1$ & 1 & 42.2 & 0.14 & 2.27 & 0.02 & 0.99939 & 28 & - \\
\hline 3 & Methanol-Aceton & $0.03-24.7$ & 1 & 42.2 & 0.10 & 2.53 & 0.02 & 0.99973 & 29 & - \\
\hline 4 & Wasser-Aceton & $0.06-55.4$ & 1 & 42.2 & 0.31 & 2.83 & 0.02 & 0.99963 & 29 & $16.5[\mathrm{k}]$ \\
\hline 5 & $N$-tert-Butylformamid-Aceton & $0.01-9.0$ & 1 & 46.0 & 0.27 & 1.87 & 0.01 & 0.99948 & 31 & - \\
\hline 6 & 1,4-Butandiol-Acetonitril & $0.01-12.5$ & 1 & 46.0 & 0.01 & 1.53 & 0.06 & 0.99749 & 31 & - \\
\hline 7 & Ethanol-Acetonitril & $0.01-17.1$ & 1 & 46.0 & 0.10 & 1.83 & 0.01 & 0.99972 & 30 & 12.0 \\
\hline 8 & 1-Hexanol-Acetonitril & $0.01-8.0$ & 1 & 46.0 & 0.08 & 1.08 & 0.02 & 0.99876 & 29 & - \\
\hline 9 & Methanol-Acetonitril & $0.03-24.7$ & 1 & 46.0 & 0.06 & 1.83 & 0.03 & 0.99877 & 31 & - \\
\hline 10 & Wasser-Acetonitril & $0.06-49.8$ & 1 & 46.0 & 0.15 & 2.07 & 0.02 & 0.99877 & 27 & - \\
\hline 11 & $N$-tert-Butylformamid-Benzol & $0.01-9.0$ & 1 & 35.2 & 0.017 & 2.42 & 0.02 & 0.99935 & 30 & - \\
\hline 12 & Wasser-tert-Butylalkohol & $0.06-33.2$ & 1 & 43.9 & 1.01 & 2.82 & 0.05 & 0.99666 & 26 & - \\
\hline 13 & Wasser-tert-Butylhydroperoxid & $0.4-7.4$ & 1 & 49.7 & 0.312 & 1.40 & 0.02 & 0.99922 & 12 & - \\
\hline 14 & Ethano!-1-Decanol & $0.02-17.1$ & 1 & 47.6 & 4.09 & 2.35 & 0.02 & 0.99915 & 30 & - \\
\hline 15 & 1-Butanol-Dimethylformamid & $0.01-10.9$ & 1 & 43.8 & 1.64 & 3.20 & 0.02 & 0.99936 & 30 & 8.7 \\
\hline 16 & Ethanol-Dimethylformamid & $0.02-17.1$ & 1 & 43.8 & 0.607 & 2.78 & 0.04 & 0.99792 & 30 & 13.7 \\
\hline 17 & Methanol-Dimethylformamid & $0.03-24.7$ & 1 & 43.8 & 0.696 & 3.55 & 0.05 & 0.99745 & 31 & - \\
\hline 18 & Wasser-Dimethylformamid & $0.06-38.8$ & 1 & 43.8 & 11.43 & 9.24 & 0.18 & 0.99527 & 27 & - \\
\hline 19 & 1-Butanol-Dimethylsulfoxid & $0.01-10.9$ & 1 & 45.0 & 4.29 & 4.93 & 0.1 & 0.99475 & 30 & 7.7 \\
\hline 20 & Ethanol-Dimethylsulfoxid & $0.02-17.1$ & 1 & 45.0 & 2.69 & 3.95 & 0.05 & 0.99794 & 30 & 13.7 \\
\hline 21 & Acetonitril-1,4-Dioxan & $0.02-19.1$ & 1 & 36.0 & 0.77 & 3.23 & 0.03 & 0.99909 & 29 & - \\
\hline 22 & 1-Butanol-1,4-Dioxan & $0.01-10.1$ & 1 & 36.0 & 0.90 & 5.39 & 0.03 & 0.99973 & 30 & - \\
\hline 23 & Ethanol-1,4-Dioxan & $0.02-17.1$ & 1 & 36.0 & 0.72 & 4.99 & 0.03 & 0.99975 & 30 & - \\
\hline 24 & Methanol-1,4-Dioxan & $0.03-3.0$ & 1 & 36.0 & 0.98 & 7.67 & 0.03 & 0.99980 & 20 & 3.0 \\
\hline 25 & Methanol-1,4-Dioxan & $3.0-24.7$ & 1 & $(32.3)[\mathrm{m}]$ & 0.089 & 4.09 & 0.08 & 0.99828 & 10 & (3.0) \\
\hline 26 & Nitromethan-1,4-Dioxan & $0.02-18.6$ & 1 & 36.0 & 1.01 & 3.49 & 0.03 & 0.99929 & 31 & - \\
\hline
\end{tabular}




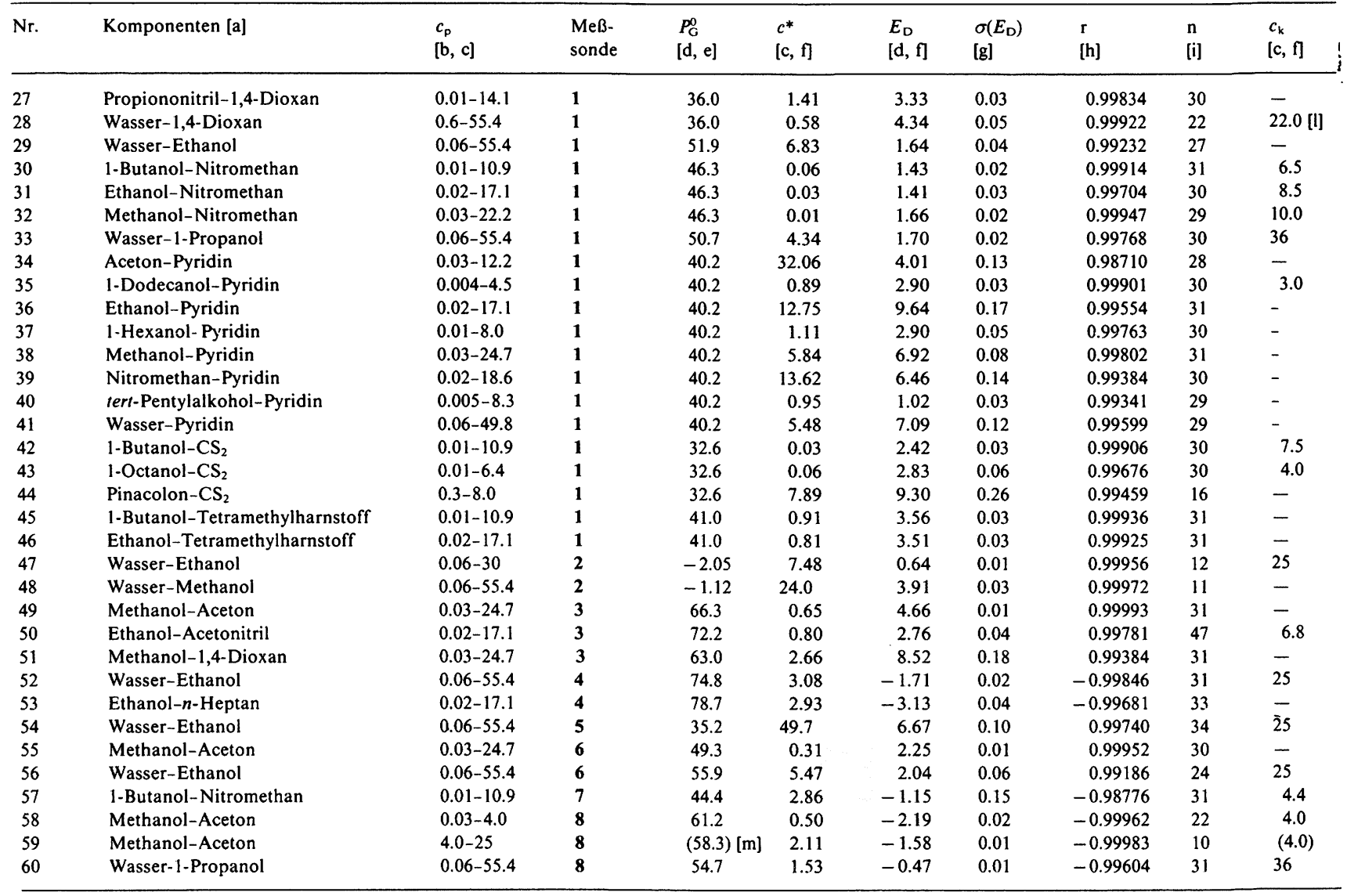

[a] Das stärker polare Lösungsmittel ist an erster Stelle aufgeführt, beide Komponenten in alphabetischer Reihenfolge. [b] Untersuchter Konzentrationsbereich. [c] In mol $\cdot \mathrm{L}^{-1}$. [d] In kcal $\cdot \mathrm{mol}^{-1}$. [e] $P_{\mathrm{G}}$-Wert der weniger polaren Komponente (siehe auch [2]). [f] Siehe Text. [g] Varianz von $E_{\mathrm{D}}$. [h] Korrelationskoeffizient bei Anwendung von Gleichung (4). [i] Anzahl der Meßpunkte. [k] Für $c_{\mathrm{p}}>c_{\mathrm{k}}$ gilt $E_{\mathrm{D}}=6.8$ und $c^{*}=6.1$. [l] Für $c_{\mathrm{p}}<c_{\mathrm{k}}$ gilt $E_{\mathrm{D}}=20.0$ und $c^{*}=19.1$. [m] Extrapolierter Wert.

Bei einer kritischen Konzentration $c_{\mathrm{k}}$ geht jedoch die lineare Beziehung abrupt in eine zweite, steilere mit anderen $E_{\mathrm{D}^{-}}$und $c^{*}$-Werten über ${ }^{[29]}$. Die Konzentrationsbereiche $c_{\mathrm{p}}<c_{\mathrm{k}}$ und $c_{\mathrm{p}}>c_{\mathrm{k}}$ werden im folgenden Bereich I und Bereich II genannt. Für das Gemisch Wasser-1,4-Dioxan läßt sich, wie in Figur 3 gezeigt, der abrupte Übergang bei $c_{k}$ (Unstetigkeitsstelle in der 1. Ableitung) bereits beim Auftragen der $E_{\mathrm{T}}(30)$-Werte gegen $\ln c_{\mathrm{p}}$ erkennen, also ohne

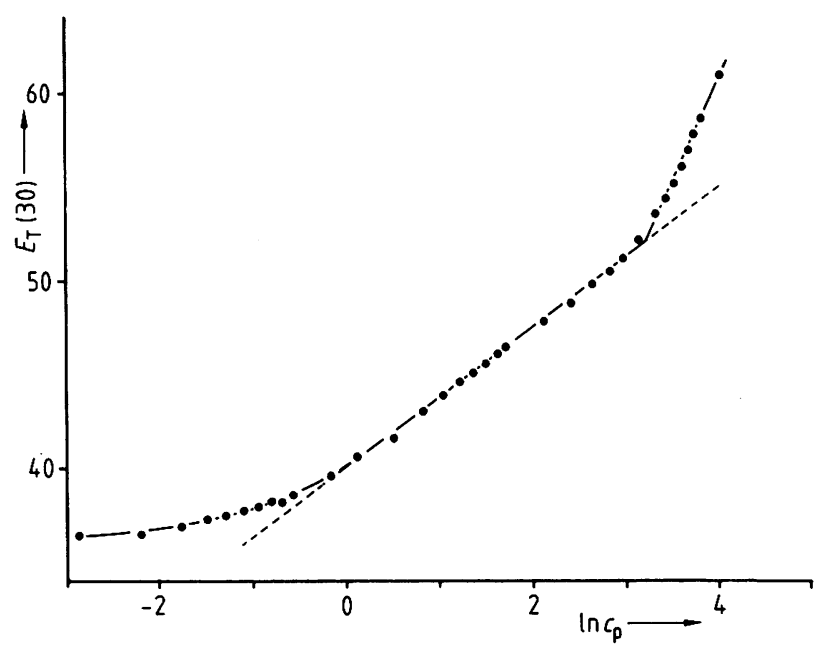

Fig. 3. Doppelgerade für das Gemisch Wasser-1,4-Dioxan (Nr. 28).
Einführen von zusätzlichen Parametern. Die Existenz zweier Gültigkeitsbereiche von Gleichung (3) mit einem kritischen Übergang bei $c_{\mathrm{k}}$ kann so interpretiert werden, $\mathrm{da} ß \mathrm{im}$ Bereich I bei kleinen Konzentrationen an Wasser noch weitgehend isolierte Wassermoleküle vorliegen, während in Bereich II der Übergang zur WasserstoffbrückenStruktur des Wassers stattfindet. In anderem Zusammenhang wurde postuliert, daß 1,4-Dioxan die Wirkung eines „solvent-structure-breaker" hat ${ }^{[39]}$. Dieses Bild stimmt gut mit den Ergebnissen der Polaritätsmessungen überein.

In Figur 3 kann der lineare Abschnitt des Bereiches $I$ in den Bereich II hinein extrapoliert werden (gestrichelte Linie; noch genauer wird das Ergebnis, wenn man statt gegen $\ln c_{\mathrm{p}}$ gegen $\ln \left(c_{\mathrm{p}} / c^{*}+1\right)$ aufträgt). Man erhält dann für $c_{\mathrm{p}}=c_{\mathrm{p}}^{\max }$ einen virtuellen Polaritätswert, $P_{\mathrm{v}}=56$, für Wasser, der kleiner als dessen tatsächliche Polarität von $63.1^{[2]}$ ist. $P_{\mathrm{v}}$ ist die Polarität, mit der das Wasser im Bereich $I$ als stärker polare Komponente auf das Dioxan wirkt. Da bei starker Verdünnung die wirksame Polarität von Wasser wesentlich geringer ist als die in konzentrierter Lösung, ließe sich spekulativ folgern, daß für die hohe Polarität von reinem Wasser weniger die Eigenschaften des isolierten Wasser-Moleküls maßgeblich sind als vielmehr dessen besondere Wechselwirkungen im Molekülverband.

Das Auftreten einer zweiten, steileren Geraden nach Gleichung (3) ist nicht auf das Gemisch Wasser-1,4-Dioxan beschränkt, sondern wird auch bei anderen Gemi- 
schen, insbesondere mit Wasser, z. B. Wasser-Aceton, beobachtet ${ }^{[40]}$. Eingehend wurde das Gemisch Wasser-Ethanol unter Anwendung verschiedener Polaritätsskalen untersucht ${ }^{[29]}$; mit allen Skalen wurden hierbei Doppelgeraden erhalten (drei Beispiele zeigt Figur 4).

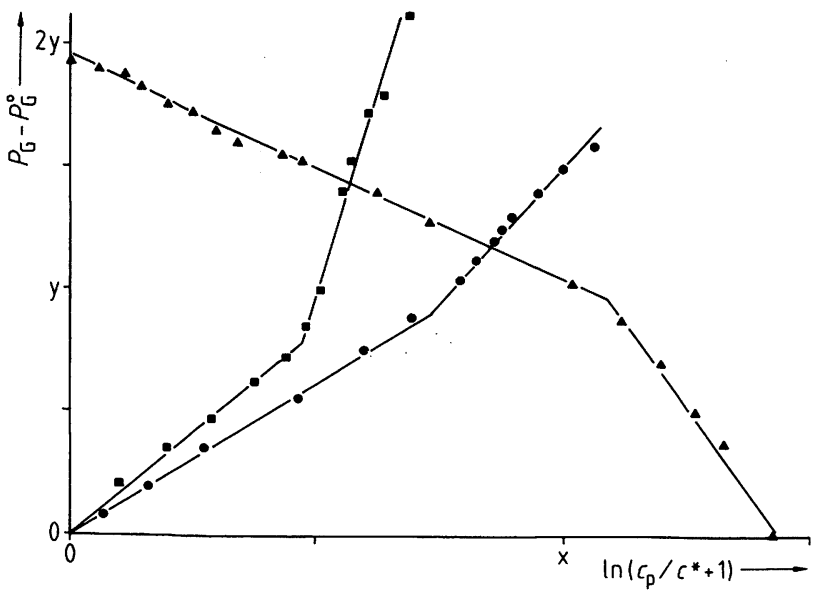

Fig. 4. Doppelgeraden nach Gleichung (4) für das Gemisch Wasser-Ethanol (Nr. 29): $Y, \mathrm{x}=2, \mathrm{y}=1 ; \square E_{\mathrm{T}}(1), \mathrm{x}=1, \mathrm{y}=4 ; \Delta \pi \neq, \mathrm{x}=2, \mathrm{y}=4$, Ordinate $P_{\mathrm{G}}-P_{\mathrm{G}}^{0}+7.7$

Bemerkenswert ist, daß bei den verschiedenen Polaritätsskalen die $c_{\mathrm{k}}$-Werte in den Grenzen der experimentellen Unsicherheiten übereinstimmen ${ }^{[*]}$. Das Phänomen der Doppelgeraden ist somit keine Besonderheit der $E_{\mathrm{T}}(30)$ Skala, sondern ist als Eigenschaft des binären Gemisches Wasser-Ethanol anzusehen. In Abschnitt 4.1 wird dieses Problem noch näher erörtert.

Interessanter noch als die zuvor behandelten Beispiele erscheinen Gemische aus 1-Butanol und Acetonitril oder Nitromethan sowie aus Ethanol und Nitromethan, die Beispiele für einen weiteren Typ binärer Gemische sind ${ }^{[31]}$. Es werden hier ebenfalls zwei Geraden beobachtet. Die zweite Gerade hat jedoch im Gegensatz zu den vorher genannten Fällen eine negative Steigung. In Figur 5 ist dies für das Gemisch 1-Butanol-Nitromethan dargestellt.

Die Polarität des Gemisches steigt mit wachsendem $c_{\mathrm{p}}$ und erreicht bei $c_{\mathrm{k}}$ ihr Maximum. Bei noch weiterem Anwachsen von $c_{\mathrm{p}}$ sinkt sie jedoch wieder und erreicht bei $c_{\mathrm{p}}^{\max }$ den Polaritätswert der reinen stärker polaren Komponente. Im Bereich $\mathrm{I}, c_{\mathrm{p}}<c_{\mathrm{k}}$, wird normales Verhalten nach Gleichung (3) beobachtet (vgl. Figur 5a). Im Bereich II jedoch wirkt das als Reinsubstanz weniger polare Nitromethan auf das 1-Butanol wie ein stärker polarer Zusatz. Entsprechend besteht in diesem Bereich ein linearer Zusammenhang zwischen $E_{\mathrm{T}}(30)$ und $\ln \left(c_{\mathrm{u}} / c^{*}+1\right)$ mit $c_{\mathrm{u}}$ als molarer Konzentration der schwächer polaren Komponente (Figur 5b). Analog zu den vorangegangenen Beispielen läßt sich hier für die polare Komponente 1-Butanol eine virtuelle Polarität definieren. Im Gegensatz zu diesen Beispielen ist sie jedoch größer als die tatsächliche Polarität des Alkohols.

Gemische mit $c_{\mathrm{p}}=c_{\mathrm{k}}$ sind wesentlich polarer als ihre Komponenten. Dieses als Polaritätsüberhöhung ${ }^{[31]}$ oder synergetischer Polaritätseffekt ${ }^{[41]}$ bezeichnete Phänomen

[*] In Figur 4 erscheinen die Knickpunkte bei verschiedenen Abszissenwerten, weil sich die $c^{*}$-Werte von Polaritätsskala zu Polaritätsskala unterscheiden. läßt sich mit einem Wasserstoffbrücken-Donor-AcceptorModell interpretieren: Der selbst nur wenig polare Zusatz forciert die Bildung einer polaren Lösungsmittelstruktur.
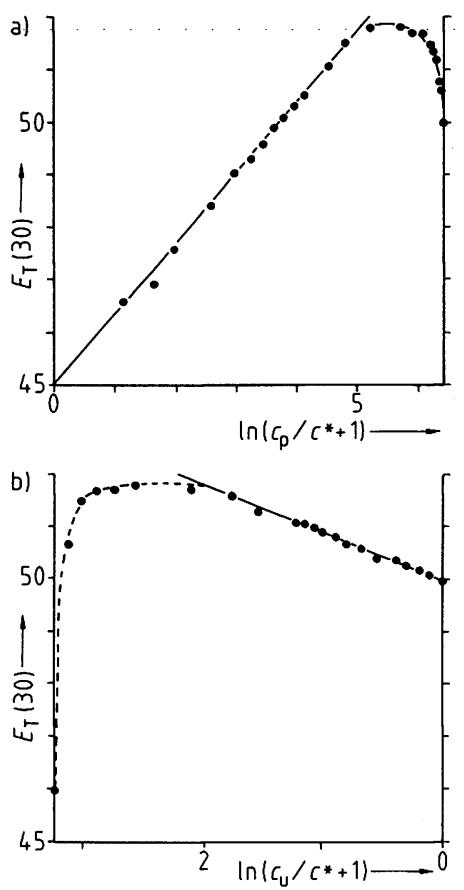

Fig. 5. Zusammenhang zwischen $E_{\mathrm{T}}(30)$ und a) $\ln \left(c_{\mathrm{p}} / c^{*}+1\right)$ sowie b) $\ln \left(c_{\mathrm{u}} / c^{*}+1\right)$ für das Gemisch 1-Butanol-Nitromethan.

Ein solches Verhalten ist insofern bemerkenswert, als es zum einen der geläufigen Anschauung widerspricht, daß die Polarität eines Lösungsmittelgemisches in den Grenzen der Polaritäten seiner Komponenten liegt, und zum anderen die Möglichkeit eröffnet, die Polarität von Solventien auch durch den Zusatz weniger polarer Komponenten zu erhöhen.

\subsection{Langsame und schnelle Prozesse}

Bei der Mehrzahl der Polaritätsskalen wird für reine Solventien eine lineare Korrelation der Skalen untereinander und mit der $Y$-sowie mit der $E_{\mathrm{T}}(30)$-Skala gefunden. Eine Ausnahme hiervon scheinen einige wenige Skalen zu bilden, die wiederum untereinander korrelieren. Typischerweise wurde dieses Verhalten bei schwach polaren Merocyaninen mit positiver Solvatochromie festgestellt ${ }^{[6,42]}$. Eines der als erste untersuchten Beispiele ist die $\chi_{\mathrm{R}}$-Skala (Tabelle 1, Nr. 7) von Brooker et al. ${ }^{[13]}$, die auf der Solvatochromie von Farbstoff 7 basiert. Die Polaritätsskalen wie $\chi_{\mathrm{R}}$ sollen hier besonders im Hinblick auf ihre Anwendung bei binären Flüssigkeitsgemischen diskutiert werden.

Phthalimidderivate 8 zeigen eine stark positive Solvatochromie in der Fluoreszenz, die von Zelinski et al. ${ }^{[35,36]}$ als Basis für die $S$-Polaritätsskala vorgeschlagen worden ist (zur Einwirkung von Sauerstoff vgl. ${ }^{[37]}$ ). Aber auch die Absorption dieser Farbstoffe erweist sich als solvatochrom $^{[30]}$. Einerseits gibt es bei diesem System einen linearen Zusammenhang zwischen Fluoreszenz und $E_{\mathrm{T}}(30)$-Skala, andererseits konnte nun ein solcher zwischen Absorp- 
tion und $\chi_{\mathrm{R}}$-Skala beobachtet werden. Farbstoff 8 ist daher zur Klärung der vorher erwähnten Ausnahmen besonders geeignet, da er beide Gruppen von Polaritätsskalen unter einen Hut zu bringen vermag.

Es ist bekannt, daß der Grundzustand $\left(S_{0}\right)$ von 8 nur ein kleines Dipolmoment hat, der erste elektronisch angeregte Zustand $\left(S_{1}\right)$ hingegen ein großes ${ }^{[7]}$. Nimmt man an, daß Zustände mit großem Dipolmoment von einem polaren Solvens stark solvatisiert werden, Zustände mit kleinem Dipolmoment aber nur schwach, so läßt sich das unterschiedliche Verhalten von 8 in Absorption und Emission erklären: Das Franck-Condon-Prinzip für einen Elektronenübergang bezieht die Solvathülle eines Farbstoffs mit ein $^{[43]}$. Der Elektronenübergang vollzieht sich in so kurzer Zeit, daß ihm die Solvatation durch Ausrichtung der Solvensmoleküle nicht folgen kann. Deshalb wird sich eine Solvatation durch Ausrichtung (Orientierung) nur auf den Ausgangszustand eines Elektronenübergangs auswirken, nicht jedoch auf den Endzustand. Dieser trägt noch die Solvathülle des Ausgangszustandes. Der Endzustand kann nur durch Polarisierungseffekte stabilisiert werden.

Absorption und Fluoreszenz von $\mathbf{8}$ laufen nach dem in Figur 6 angegebenen Schema ab. Der Ausgangszustand, $S_{0}$, wird nur wenig von polaren Solventien beeinflußt.

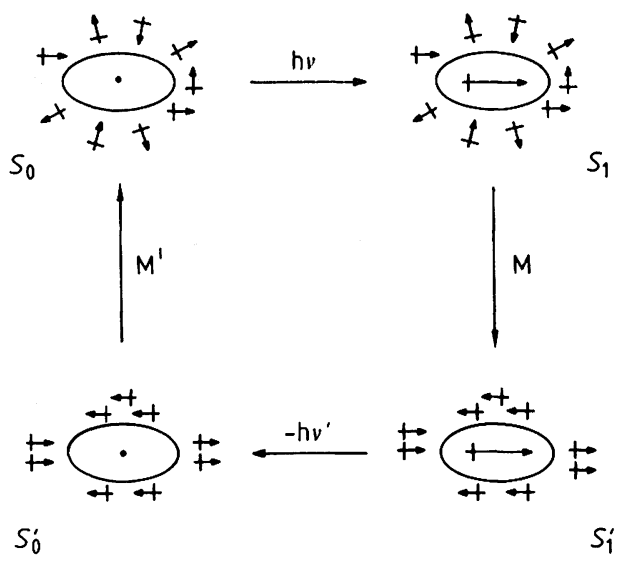

Fig. 6. Schematische Darstellung der Absorptions- und Emissionsvorgänge bei Farbstoff 8 in polaren Solventien.

Der angeregte Zustand, $S_{1}$, hat zwar ein großes Dipolmoment, trägt aber noch die Solvathülle des $S_{0}$-Zustandes. Auf die Absorption wirken sich also vorwiegend SolvensPolarisierungseffekte aus - analog bei der $\chi_{\mathrm{R}}$-Skala. An die Anregung schließt sich die Relaxation der Solvathülle an; die Lebensdauer des $S_{1}$-Zustandes ist mit $10^{-8}$ bis $10^{-9} \mathrm{~s}$ groß genug, um den Solvensmolekülen in der Umgebung des Farbstoffs eine günstigere Orientierung zu ermöglichen. Dieser Übergang von $S_{1}$ nach $S_{1}^{\prime}$ entspricht der Modifizierung (M), wie sie für Reaktionen mit angeregten $\mathrm{Zu}$ ständen beschrieben wurde ${ }^{[44]}$, bei denen die Anregungsenergie erhalten bleibt. Die Fluoreszenz ist schließlich der Übergang von $S_{1}^{\prime}$ nach $S_{0}^{\prime}$, auf dessen Energie sich SolvensOrientierungseffekte (neben Polarisierungseffekten) voll auswirken, weil der Ausgangszustand dieses Elektronenübergangs ein großes Dipolmoment hat. Es wird daher verständlich, daß die Fluoreszenz von $8 \mathrm{mit} \operatorname{der} E_{\mathrm{T}}(30)$ Skala korreliert. Analog ist die Situation für Farbstoff 1 bei der Absorption. In den meisten Fällen sind die Ver- hältnisse aber nicht so extrem wie bei den Farbstoffen 7 oder $\mathbf{8}$ in Absorption, und man beobachtet eine Mischung von Polarisierungs- und Orientierungseffekten. Häufig überwiegen die Orientierungsphänomene, woraus eine Korrelation mit der $E_{\mathrm{T}}(30)$-Skala resultiert.

So wird deutlich, daß Farbstoff $7\left(\chi_{\mathrm{R}}\right.$-Skala) eine Polaritätssonde mit kurzer Meßzeit, Farbstoff 1 ( $E_{\mathrm{T}}(30)$-Skala) jedoch eine solche mit langer Meßzeit ist (vgl. auch das Konzept von Bakhshiev ${ }^{[45]}$ ). In der Organischen Chemie ist für viele Prozesse die Orientierung des Solvens von entscheidendem Einfluß, also ein verhältnismäßig langsamer Vorgang. Dies erklärt, warum gerade auf diese Prozesse die $E_{\mathrm{T}}(30)$-Skala zutrifft.

Gleichung (4) beschreibt die Polarität binärer Flüssigkeitsgemische nach der $\chi_{R}$-Skala oder einer AbsorptionsSkala mit Farbstoff 8 als Meßsonde. Daher sollte diese Gleichung auch das polare Verhalten von binären Lösungsmittelgemischen bei schnellen Prozessen wiedergeben. Ihre Gültigkeit ist keinesfalls allein auf eine Anreicherung des polaren Solvens an der Oberfläche des Farbstoffs zurückzuführen ${ }^{[46,47]}$. Informationen über die SolvensStruktur liefert ein Vergleich der $c^{*}$-Werte für Solvensgemische mit Farbstoff 8 in Absorption und Emission ${ }^{[30]}$.

\section{Anwendungen der allgemeinen Gleichung (4)}

Da Gleichung (4) das polare Verhalten von binären Flüssigkeitsgemischen quantitativ beschreibt, mangelt es nicht an Gelegenheiten, sie als „Werkzeug“ in verschiedenen Bereichen der Chemie zu benutzen. Zunächst werden Anwendungen auf mechanistische Probleme behandelt.

\subsection{Die Solvolyse von tert-Butylchlorid in Ethanol-Wasser}

Das Auffinden der Winstein-Beziehung ${ }^{[9]}$, die auf der Solvolysereaktion von tert-Butylchlorid basiert, spielte eine Schlüsselrolle bei der Entwicklung des empirischen Polaritätsbegriffs. Winstein untersuchte auch den Temperatureinfluß auf die Solvolyse von tert-Butylchlorid in Ethanol-Wasser-Gemischen ${ }^{[1]}$. Während die Freie Aktivierungsenthalpie $\Delta G^{+}$dieser Reaktion als monotone Funktion ohne starke Krümmung mit dem Wassergehalt der Mischung abnimmt, treten für $\Delta H^{+}$und $\Delta S^{+}$Maxima und Minima auf (Fig. 7).

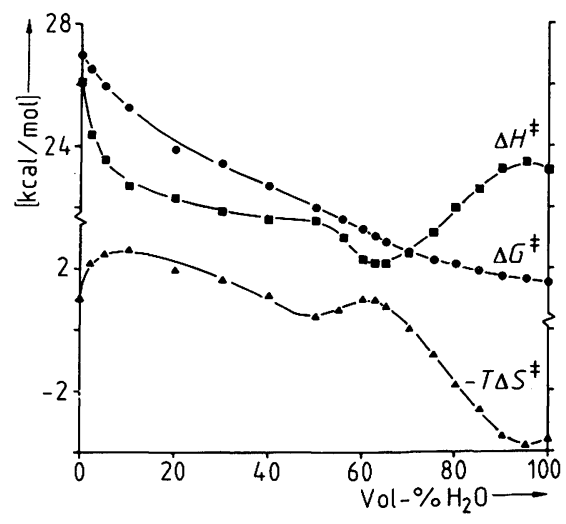

Fig. 7. Thermodynamische Daten der Solvolyse von tert-Butylchlorid in Ethanol-Wasser [11]. 
Dieses ungewöhnliche und überraschende Verhalten hat

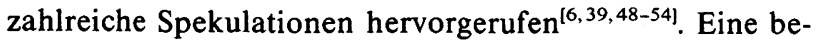
friedigende Erklärung der vor 25 Jahren veröffentlichten experimentellen Befunde steht jedoch bis heute aus. Der Erfahrung scheint zu widersprechen, daß die aus enthalpischen und entropischen Termen zusammengesetzte Größe $\Delta G^{\ddagger}$ einen einfachen Funktionsverlauf zeigt, die anscheinend einfacheren Größen $\Delta H^{*}$ und $\Delta S^{+}$aber einen komplexen.

Da die Solvolyse von tert-Butylchlorid empfindlich auf Polaritätsänderungen des Mediums anspricht, bietet sich eine Analyse der Meßergebnisse von Winstein und Fainberg unter Anwendung von Gleichung (4) an. Wie Figur 8 demonstriert, lassen sich die Freie Aktivierungsenthalpie $\Delta G_{298}^{+}$der Solvolyse von tert-Butylchlorid in Ethanol-Wasser-Gemischen und die dazu proportionalen Größen $\ln k_{298}$ oder $Y$ als Funktion der Zusammensetzung quantitativ mit Gleichung (4) beschreiben. Als Besonderheiten werden wie auch bei anderen Polaritätsskalen (vgl. Abschnitt 3.1.3) - zwei Gültigkeitsbereiche von Gleichung (4) mit einem kritischen Übergang bei $c_{\mathrm{k}} \approx 25 \mathrm{~mol} \cdot \mathrm{L}^{-1}$ gefunden (Doppelgeraden). Die Freie Aktivierungsenthalpie zeigt somit keinerlei Anomalien. Hervorzuheben ist die hohe Präzision der kinetischen Messungen von Winstein et al., die in den Geraden von Figur 8 und Figur 2 voll zum Ausdruck kommt.

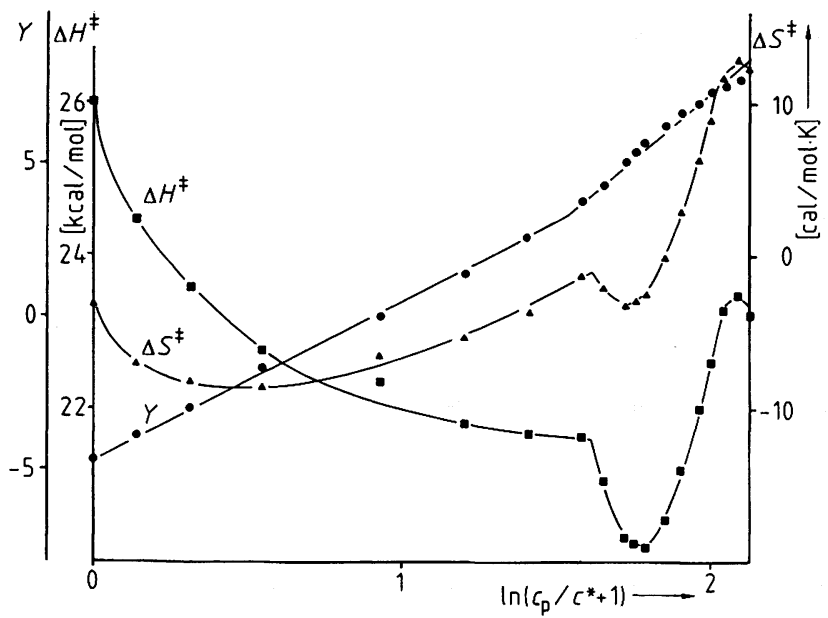

Fig. 8. Analyse der Solvolysedaten von tert-Butylchlorid in Ethanol-Wasser anhand von Gleichung (4).

Die Aktivierungsenthalpie $\Delta H^{\ddagger}$ und die Aktivierungsentropie $\Delta S^{+}$der Reaktion sind aus den Geschwindigkeitskonstanten bei nur jeweils zwei Temperaturen berechnet worden ${ }^{[1]}$, im Bereich von 0 bis 45 Vol.-\% Wasser bei 25 und $50^{\circ} \mathrm{C}$, im Bereich von 50 bis 100 Vol.- $\%$ Wasser 0 und $25^{\circ} \mathrm{C}$. Auch die Meßreihen bei 0 und $50^{\circ} \mathrm{C}$ entsprechen Gleichung (4) (siehe Tabelle 3 ); ihre $E_{\mathrm{D}^{-}}$und $c^{*}$ Werte unterscheiden sich nur wenig von den Werten bei $25^{\circ} \mathrm{C}$.

Der Temperaturwechsel von $0^{\circ} \mathrm{C}$ zu $50^{\circ} \mathrm{C}$ für die Berechnung der Aktivierungsdaten wurde bei einer Wasserkonzentration von ca. $25 \mathrm{~mol} \cdot \mathrm{L}^{-1}$ vorgenommen (die zweite Temperatur beträgt stets $25^{\circ} \mathrm{C}$ ); dieser Wert stimmt zufällig ungefähr mit dem Wert von $c_{\mathrm{k}}$ überein, der dem Schnittpunkt der beiden Geraden in Figur 8 entspricht. Auf dieser Basis läßt sich der Kurvenverlauf von $\Delta H^{\ddagger}$ in Figur 7 beschreiben.
Tabelle 3. Anwendung von Gleichung (4) auf die Winstein-Skala.

\begin{tabular}{clccccc}
\hline$T$ [a] & Bereich [b] & $E_{\text {D }}$ [c] & $c^{*}[\mathrm{~d}]$ & $Y^{0}$ [e] & r [f] & n [g] \\
\hline 25 & I & 2.9 & 11.6 & -1.97 & 0.9991 & 9 \\
50 & I & 2.4 & 9.7 & -0.52 & 0.9998 & 7 \\
0 & II & 2.6 & 0.001 & $(-26.9)$ & 0.9845 & 12 \\
25 & II & 2.7 & 0.001 & $(-26.1)$ & 0.9935 & 10 \\
$\left(\Delta H^{+}\right)[\mathrm{b}]$ & I & $-0.944[\mathrm{i}]$ & 0.26 & $(26.1)[\mathrm{h}, \mathrm{i}]$ & 0.9996 & 6 \\
\hline
\end{tabular}

[a] Temperatur in ${ }^{\circ} \mathrm{C}$. [b] Siehe Text. [c] In $Y$-Einheiten. [d] mol $\cdot \mathrm{L}^{-1}$. [e] Extrapolierter $Y$-Wert für Ethanol. [f] Korrelationskoeffizient. [g] Anzahl der Meßwerte. [h] Aktivierungsenthalpie. [i] In $\mathrm{kcal} \cdot \mathrm{mol}^{-1}$.

Für $c_{\mathrm{p}}<c_{\mathrm{k}}$ (Bereich I, es gilt $\Delta H^{+} \sim \ln k_{25}{ }^{\circ} \mathrm{C}-\ln k_{50}{ }^{\circ} \mathrm{C}$ ) ist $\Delta H^{+}$eine monotone Funktion des Wassergehalts. Bei den beiden Temperaturen unterscheiden sich die $E_{\mathrm{D}^{-}}$und $c^{*}$ Werte von $\ln k$ nur wenig. Es wird daher sogar die SolvensAbhängigkeit von $\Delta H^{\ddagger}$ näherungsweise durch Gleichung (4) beschrieben (Tabelle 3). Bei dieser Korrelation ist noch die thermische Ausdehnung der Flüssigkeit zu berücksichtigen, da bekanntlich bei einem bestimmten Gehalt in Vol.$\%$ der molare Wassergehalt temperaturabhängig ist.

Bei $c_{\mathrm{p}} \approx c_{\mathrm{k}}$ kommen mehrere Effekte zusammen, die die Konzentrationsabhängigkeit von $\Delta H^{+}$komplizieren. Das Überschreiten von $c_{\mathrm{k}}$, dessen Temperaturabhängigkeit und der Temperaturwechsel bewirken, daß die Einzelmessungen zur Bestimmung von $\Delta H^{+}$teilweise in Bereich I und in Bereich II ausgeführt wurden. Daraus resultiert der ungewöhnliche Funktionsverlauf von $\Delta H^{*}$.

Für $c_{\mathrm{p}}>c_{\mathrm{k}}$ (Bereich II) wird schließlich wie im Bereich I eine monotone Abhängigkeit der $\Delta H^{+}$-Werte von der Lösungsmittelzusammensetzung beobachtet. Das weitere Maximum bei sehr hohem Wassergehalt könnte auf Mischungsprobleme zurückzuführen sein (vgl. dazu ${ }^{[48,55]}$ ). Die Konzentrationsabhängigkeit von $\Delta S^{*}$ (siehe die Figuren 7 und 8) läßt sich in analoger Weise als Differenz von $\Delta H^{*}$ und $\Delta G^{*}$ diskutieren.

Die hier ausführlich behandelte Solvolyse von tert-Butylchlorid in Ethanol-Wasser hat Modellcharakter für andere Solvolysereaktionen in Solvens-Gemischen ${ }^{[39.52 .53,56-59]}$, die analog behandelt werden können, und auch allgemein für mechanistische Untersuchungen in Solvens-Gemischen. Weiterhin könnte der Bezugspunkt der $Y$-Skala: 80 Vol.-\% Ethanol/Wasser bei Messungen problematisch werden, wenn die Temperatur stark von $25^{\circ} \mathrm{C}$ abweicht. Noch ein anderes Resultat unserer Analyse ist von allgemeinerem Interesse: Bei Reaktionen, die von der Polarität des Mediums beeinflußt werden, sollte zumindest in Solvens-Gemischen die Freie Aktivierungsenthalpie $\Delta G^{+}$eine einfachere Größe sein als $\Delta H^{+}$und $\Delta S^{+}$, da die letzteren immer Beiträge von der thermischen Ausdehnung der Flüssigkeit enthalten, die nicht reaktionsspezifisch sind. Besondere Aufmerksamkeit ist außerdem geboten, wenn ein Solvens-Gemisch zwei Gültigkeitsbereiche von Gleichung (4) aufweist (vgl. auch Abschnitt 4.2).

\subsection{Nichtlineare Winstein-Beziehungen}

Die zuletzt genannten Ergebnisse führen zu weiteren Anwendungen von Gleichung (4) bei mechanistischen Untersuchungen: Eine lineare Korrelation eines Prozesses mit der Winstein-Skala wird vielfach analog zu anderen linea- 
ren Beziehungen zwischen Freien Enthalpien (LFE-Beziehungen) als mechanistisches Kriterium verwendet ${ }^{[32,60-64]}$. So wird die Größe $m$, die Steigung der Geraden, als Maß für die Empfindlichkeit interpretiert, mit der die zu untersuchende Reaktion auf Solvens-Polaritätseffekte anspricht, und oft mit der Zunahme oder Abnahme von Ladungstrennung bei der Bildung des Übergangszustandes in Beziehung gesetzt. Andererseits wird eine lineare Korrelation über einen großen Solvens-Polaritätsbereich als Kriterium für einen einheitlichen Reaktionsmechanismus auch bei Veränderung des Lösungsmittels angesehen. Um genügend Meßpunkte zu erhalten, werden die Reaktionen meist in Solvens-Gemischen untersucht, häufig in Ethanol-Wasser.

Da die Polarität dieser Gemische Gleichung (4) folgt, erscheint es lohnend, die erwähnten mechanistischen Kriterien auf der Basis von Gleichung (4) neu zu formulieren.

Aus Gleichung (4) ergibt sich für die Steigung $m$ einer Beziehung zwischen zwei polaritätsabhängigen Prozessen $P_{\mathrm{G} 1}$ und $P_{\mathrm{G} 2}$ die Gleichung (5).

$m=\frac{\mathrm{d} P_{\mathrm{G} 1}}{\mathrm{~d} P_{\mathrm{G} 2}}=\frac{E_{\mathrm{D} 1}\left(c_{\mathrm{p}}+c_{2}^{*}\right)}{E_{\mathrm{D} 2}\left(c_{\mathrm{p}}+c_{1}^{*}\right)}$

Für eine gute lineare Beziehung zwischen $P_{\mathrm{G} 1}$ und $P_{\mathrm{G} 2}$ muß die Steigung $m$ in Gleichung (5) unabhängig von $c_{\mathrm{p}}$ sein und somit eine der drei folgenden Bedingungen erfüllt werden:

1. Die $c^{*}$-Werte sind bei beiden Prozessen ähnlich $\left(c_{1}^{*} \approx c_{2}^{*}\right)$. Der logarithmische Ausdruck in Gleichung (4) erhält dann jeweils für ein bestimmtes $c_{\mathrm{p}}$ in beiden Fällen etwa den gleichen numerischen Zahlenwert. $P_{\mathrm{G} 1}$ hängt folglich linear von $P_{\mathrm{G} 2}$ ab.

2. Die $c^{*}$-Werte sind bei beiden Prozessen unterschiedlich, aber wesentlich größer als $c_{\mathrm{p}}^{\text {max }}\left(c_{1}^{*}, c_{2}^{*} \gg c_{\mathrm{p}}^{\max }\right)$. Gleichung (4) läßt sich in beiden Fällen als Taylor-Reihe entwikkeln, die wie üblich abgebrochen wird. Es resultiert jeweils eine lineare Beziehung zwischen $P_{\mathrm{G} 1}$ und $c_{\mathrm{p}}$ und damit auch eine lineare Beziehung zwischen $P_{\mathrm{G} 1}$ und $P_{\mathrm{G} 2}$.

3. Die $c^{*}$-Werte sind unterschiedlich, aber beide sehr klein. $P_{\mathrm{G}}$ hängt dann jeweils linear von $\ln c_{\mathrm{p}} \mathrm{ab}$, und damit besteht auch ein linearer Zusammenhang zwischen $P_{\mathrm{G} 1}$ und $P_{\mathrm{G} 2}$.

Sehr oft ist eine der drei Bedingungen erfüllt. Wird jedoch eine nichtlineare Beziehung z. B. mit der WinsteinSkala erhalten, so folgt daraus nicht unbedingt, daß ein Wechsel im Mechanismus vorliegt, oder daß sogar ein kontinuierlicher Übergang von einem Mechanismus zu einem anderen durch die Veränderung des Mediums bewirkt wird (vgl. auch ${ }^{[65]}$ ). Hierüber kann erst durch die Bestimmung der $c^{*}$-Werte Aufschluß erhalten werden.

Komplizierter noch ist der Solvens-Einfluß und seine Abhängigkeit von $c_{\mathrm{p}}$ bei Gemischen mit kritischen Übergängen (Doppelgeraden). Diese werden insbesondere bei den für die Untersuchung von Solvolysereaktionen häufig verwendeten binären Gemischen Aceton-Wasser, 1,4-Dioxan-Wasser und Ethanol-Wasser beobachtet (vgl. Tabelle 2$)^{[29,40]}$. Ein Beispiel für das Gemisch Ethanol-Wasser soll hier behandelt werden.
Brown et al. ${ }^{[66]}$ haben die Solvolyse von Isopropyl-, Cyclohexyl- und 2-Adamantyltosylat in Ethanol-Wasser untersucht. Bis zu einem Wassergehalt von ca. $50 \%$ zeigen die Freien Aktivierungsenthalpien der Reaktionen gute lineare Korrelationen mit der $Y$-Skala. Bei höherem Wassergehalt treten größere Abweichungen auf, die einen regelrechten Knick in der LFE-Beziehung hervorrufen (Fig. 1 in ${ }^{[66]}$ ). Eine nucleophile Solvens-Beteiligung mit einem Wechsel im Reaktionsmechanismus wird diskutiert. Dem scheint jedoch zu widersprechen, daß das nur wenig abgeschirmte und einem nucleophilen Angriff leicht zugängliche Isopropyltosylat die geringsten Abweichungen zeigt. Die Abweichungen wurden schließlich einer spezifischen Solvatation des austretenden Anions zugeschrieben, da die entsprechenden Mesylate lineare Beziehungen mit der Y-Skala ergeben.

Die Solvolysedaten lassen sich auf der Basis von Gleichung (4) analysieren. Der Knick in der LFE-Beziehung wird bei einem Wassergehalt von ca. 50\% gefunden und ist als kritischer Übergang zu interpretieren (vgl. Abschnitt 3.1.3). Da für die Solvolyse von tert-Butylchlorid und der verschiedenen Tosylate jeweils ähnliche $c^{*}$-Werte erhalten werden (siehe Tabelle 4), genügt eine Diskussion der $E_{\mathrm{D}^{-}}$ Werte nach Gleichung (5). Für das tert-Butylchlorid und das Cyclohexylmesylat (Tabelle 4, Nr. 1 und 2) ist das Verhältnis der $E_{\mathrm{D}}$-Werte im Bereich I und im Bereich II fast gleich. Daraus ergibt sich in guter Näherung eine lineare LFE-Beziehung über den gesamten Konzentrationsbereich an Wasser. Der relativ etwas zu geringe $E_{\mathrm{DII}}$-Wert von $\mathrm{Nr}$. 2 erklärt sogar die geringfügigen Abweichungen bei hohem Wassergehalt.

Tabelle 4. Solvolysen $[11,66]$ im Lösungsmittelgemisch Ethanol-Wasser be $25^{\circ} \mathrm{C}$.

\begin{tabular}{|c|c|c|c|c|c|c|c|}
\hline \multirow[t]{2}{*}{ Nr. } & \multirow[t]{2}{*}{ Substrat } & \multicolumn{3}{|c|}{ Bereich I } & \multicolumn{3}{|c|}{ Bereich II } \\
\hline & & $\begin{array}{l}E_{\mathrm{DI}} \\
{[\mathrm{a}]}\end{array}$ & $\begin{array}{l}c^{*} \\
\text { [b] }\end{array}$ & $\begin{array}{l}\ln k_{01} \\
\text { [c] }\end{array}$ & $\begin{array}{l}E_{\text {DII }} \\
\text { [a] }\end{array}$ & $\begin{array}{l}c_{i}^{*} \\
{[\mathrm{~b}]}\end{array}$ & $\begin{array}{l}\ln k_{011} \\
\text { [c] }\end{array}$ \\
\hline 1 & tert-Butylchlorid & 6.7 & 11.6 & -16.1 & 6.3 & 0.001 & -7.16 \\
\hline 2 & Cyclohexylmesylat & 4.7 & 10.8 & -17.3 & 4.6 & $(0.001)[d]$ & - \\
\hline 3 & 2-Adamantyltosylat & 4.7 & 8.3 & -21.6 & 9.9 & 0.001 & -11.7 \\
\hline 4 & Isopropyltosylat & 3.2 & 12.9 & -14.7 & 5.0 & $(0.001)[d]$ & - \\
\hline 5 & Cyclohexyltosylat & 8.3 & 32.8 & -16.8 & 7.7 & 0.001 & -9.1 \\
\hline
\end{tabular}

[a] In $\ln k_{298}$-Einheiten. [b] In $\mathrm{mol} \cdot \mathrm{L}^{-1}$. [c] Aus Gleichung (4) extrapolierter Wert für reinen Alkohol. [d] Abgeschätzt, da für eine Berechnung zu wenige Meßwerte bekannt.

Ganz anders verhalten sich jedoch die Tosylate (Tabelle 4, Nr. 1, 3 und 4). Die wesentlich höheren $E_{\mathrm{D}}$-Werte im Bereich II bewirken, daß die Steigungen der LFE-Beziehungen im Bereich II fast doppelt so groß sind wie im Bereich I. Bei $c_{\mathrm{k}}$ müssen daher Knickstellen in den linearen Beziehungen auftreten (Fig. 1 in ${ }^{[66]}$ ). Beim Cyclohexyltosylat (Tabelle 4 , Nr. 1 und 5) stimmt der $c^{*}$-Wert nicht mehr so gut mit dem von Nr. 1 überein. Die LFE-Beziehung ist dementsprechend auch deutlich schlechter. Ein einfacher Vergleich der $E_{\mathrm{D}}$-Werte genügt in diesem Fall nicht mehr. Die genaue Analyse zeigt aber, daß sich Nr. 5 wie Nr. 3 und Nr. 4 verhält. Die Solvolysereaktionen in AcetonWasser-Gemischen lassen sich völlig analog interpretieren.

Von Sridharan und Vitullo ${ }^{[64]}$ wurde die Solvolyse von geminalen Dihalogeniden in 1,4-Dioxan-Wasser unter- 
sucht. Sie fanden bei der LFE-Beziehung mit der $Y$-Skala ebenfalls eine Nichtlinearität (zwei Geraden). Dies wurde als Wechsel des Reaktionsmechanismus und Übergang zu nucleophiler Solvens-Beteiligung gedeutet. Es läßt sich jedoch zeigen, daß am Schnittpunkt der beiden Geraden der kritische Übergang des Gemisches 1,4-Dioxan-Wasser vorliegt.

Die beiden zuletzt besprochenen Beispiele lassen erkennen, daß die Brauchbarkeit einer linearen Freie-EnthalpieBeziehung als mechanistisches Kriterium bei der Untersuchung solvensabhängiger Prozesse in Lösungsmittelgemischen eingeschränkt ist. Bei diesen Prozessen sollte daher in binären Gemischen auf kritische Übergänge geprüft werden.

\subsection{Weitere Anwendungen}

Mit Gleichung (4) gelingt es auch, aus polaritätsabhängigen Phänomenen Rückschlüsse auf die Zusammensetzung binärer Flüssigkeitsgemische zu ziehen ${ }^{[28,40,67]}$. Die Gemische können auf diese Weise analysiert werden. Als Meßsonden sind vor allem solvatochrome Farbstoffe geeignet. Besonders genau läßt sich damit Wasser in organischen Lösungsmitteln bestimmen, da Wasser wesentlich polarer ist als die meisten organischen Flüssigkeiten. Das Problem nichtlinearer Eichkurven mit starker Krümmung, die viele Stützpunkte im Bereich kleiner Konzentrationen an Wasser ${ }^{[68]}$ erforderten, stand einer praktischen Anwendung bisher im Wege. Gleichung (4) ermöglicht es nun, diese Eichkurven zu linearisieren und so mit wenigen Stützpunkten auszukommen. Darüber hinaus bietet der quasi-logarithmische Zusammenhang nach Gleichung (4) den Vorteil, daß der relative Meßfehler über einen großen Konzentrationsbereich annähernd konstant ist ${ }^{[40]}$. Für eine überschlägige Wasserbestimmung reicht sogar der visuelle Vergleich mit einer Farbskala aus. Lösungsmittel wie Dimethylformamid oder Dimethylsulfoxid sind hier im Gegensatz zu anderen Verfahren nicht störend. Farbige Lösungen lassen sich analog mit solvatochromen Fluoreszenzfarbstoffen untersuchen. Eine Drei-Punkt-Meßmethode macht sogar ein Fluoreszenzspektrometer entbehr$\operatorname{lich}^{[67]}$.

Weiterhin kann anhand von Gleichung (4) die Polarität einer Lösung genau eingestellt werden. Das ist von Interesse, wenn eine Verbindung in unpolaren und polaren Medien nach unterschiedlichen Mechanismen reagiert. Ein Beispiel ist die Thermolyse von Percarbonsäureestern ${ }^{[69]}$, die in unpolarer Lösung unter Homolyse und Radikalbildung verläuft, in polarer Lösung jedoch unter Heterolyse nach dem Criegee-Mechanismus. Wird eine Radikalerzeugung in einem polaren Medium gewünscht, so ist es vorteilhaft, die Polarität so hoch einzustellen, daß die Homolyse gerade noch dominiert.

In anderen Fällen, in denen ein geringer polarer Zusatz die Lösungsmittelpolarität möglichst stark erhöhen soll ${ }^{[4]}$, erhält $c^{*}$ eine Schlüsselrolle. Für $c_{\mathrm{p}}<c^{*}$ hängt die Polarität stark von $c_{\mathrm{p}}$ ab, für $c_{\mathrm{p}}>c^{*}$ aber nur noch schwach (ungefähr logarithmischer Zusammenhang). Um mit nur geringem polarem Zusatz eine erhebliche Polaritätserhöhung zu bewirken, sollte $c_{\mathrm{p}} \approx c^{*}$ eingestellt werden.

Aus Gleichung (4) kann weiter gefolgert werden, daß ein geringer polarer Zusatz zu einem wenig polaren Solvens die Polarität wesentlich erhöhen kann, während umgekehrt ein unpolarer Zusatz zu einem polaren Solvens kaum eine Erniedrigung der Polarität herbeiführt. Diesem Ergebnis entsprechen viele empirische Befunde, z. B. auf dem Gebiet der Chromatographie, wo sich polare Zusätze viel stärker auf das Elutionsverhalten auswirken als unpolare.

Die Polymerchemie ist ein weiteres Anwendungsgebiet für Gleichung (4), die nicht nur für Flüssigkeiten, sondern sogar für Feststoffe gilt ${ }^{[70]}$. So beschreibt sie zum einen die Wirkung von polaren Komponenten bei Copolymerisationen und zum anderen die von niedermolekularen Zusätzen (Weichmachern). Zur gezielten Herstellung von Polymeren mit bestimmten Eigenschaften ist dies von Bedeutung.

\section{Ausblick}

Außer in den bereits erwähnten Bereichen der Chemie läßt sich Gleichung (4) in vielen weiteren anwenden, die in diesem Fortschrittsbericht nur gestreift werden können.

Bei der Aufklärung von Reaktionsmechanismen eröffnen sich Möglichkeiten, den Medium-Einfluß auf eine chemische Reaktion auch dann zu beschreiben, wenn sich dessen Polarität während der Umsetzung ändert. Da Gleichung (4) sogar die Polarität reiner Alkohole als Funktion ihrer Kettenlänge beschreibt ${ }^{[71]}$, ist zu erwarten, daß sich die Polaritäten der Reinsubstanzen aus Inkrementen zusammensetzen lassen. Die Zusammenhänge sind jedoch nichtlinear.

In der Polymerchemie ist es nicht nur möglich, unter Verwendung von Gleichung (4) eine definierte Polarität des Polymermaterials einzustellen, polare Zusätze gezielt zu finden, die bereits in geringer Konzentration stark polaritätserhöhend wirken, und Aufschluß über die Wirkung von Rest-Monomergehalten ${ }^{[70]} \mathrm{zu}$ bekommen, sondern darüber hinaus sollte es gelingen, Informationen über Copolymerisationsparameter und über die Wechselwirkung zwischen Polymeren zu erhalten.

Schließlich haben erste Untersuchungen an ternären Flüssigkeitsgemischen ergeben, daß sich Gleichung (4) auch für Mehrkomponentensysteme erweitern läßt.

Der Deutschen Forschungsgemeinschaft sowie dem Bundesministerium für Forschung und Technologie sei für die Unterstützung der Arbeiten gedankt, meinem Lehrer Prof. Dr. C. Rüchardt für stetige Förderung. Die Berechnungen wurden mit dem Rechenprogramm POLAR am Rechenzentrum der Universität Freiburg ausgeführt.

Eingegangen am 12. August 1980, in erweiterter Form am 12. Juli 1982 [A 428]

[1] C. Reichardt: Solvent Effects in Organic Chemistry, 1. Aufl., Verlag Chemie, Weinheim 1979.

[2] C. Reichardt, Angew. Chem. 91 (1979) 119; Angew. Chem. Int. Ed. Engl. 18 (1979) 98.

[3] C. Reichardt: Lösungsmitteleffekte in der organischen Chemie, 2. Aufl., Verlag Chemie, Weinheim 1973.

[4] C. K. Ingold: Structure and Mechanism in Organic Chemistry, Cornell University Press, Ithaca, NY 1953, S. 347-350.

[5] A. Streitwieser Jr., Chem. Rev. 56 (1956) 620.

[6] K. Schwetlick: Kinetische Methoden zur Untersuchung von Reaktionsmechanismen, Deutscher Verlag der Wissenschaften, Berlin 1971, S. 143174. 
[7] I. A. Koppel, V. A. Palm in N. B. Chapman, J. Shorter: Advances in Linear Free Energy Relationships, Plenum, London 1973, S. 203-280.

[8] S. Brownstein, Can. J. Chem. 38 (1960) 1590.

[9] S. Winstein, E. Grunwald, H. W. Jones, J. Am. Chem. Soc. 73 (1951) 2700.

[10] A. H. Fainberg, S. Winstein, J. Am. Chem. Soc. 78 (1956) 2770

[11] S. Winstein, A. H. Fainberg, J. Am. Chem. Soc. 79 (1957) 5937.

[12] E. M. Kosower, J. Am. Chem. Soc. 80 (1958) 3253

[13] L. G. S. Broker, G. H. Heyes, D. W. Heseltine, J. Am. Chem. Soc. 73 (1951) 5350

[14] K. Dimroth, C. Reichardt, T. Siepmann, F. Bohlmann, Liebigs Ann Chem. 661 (1963) 1.

[15] K. Dimroth, C. Reichardt, Top. Curr. Chem. 11 (1968) 1.

[16] V. Gutmann: Coordination Chemistry in Non-Aqueous Solvents, Springer-Verlag, Wien 1968; Coord. Chem. Rev. 18 (1976) 225.

[17] M. J. Kamlet, J. L. Abboud, R. W. Taft, J. Am. Chem. Soc. 99 (1977) 6027 und zit. Lit.

[18] M. J. Kamlet, R. W. Taft, J. Org. Chem. 47 (1982) 1734 und zit. Lit.

[19] V. A. Palm: Grundlagen der quantitativen Theorie organischer Reaktionen, Akademie-Verlag, Berlin 1971.

[20] R. D. Cramer, J. Am. Chem. Soc. 102 (1980) 1849.

[21] R. D. Cramer, J. Am. Chem. Soc. 102 (1980) 1837.

[22] A. Streitwieser Jr., Chem. Rev. 56 (1956) 620.

[23] H. Langhals, E. Fritz, I. Mergelsberg, Chem. Ber. 113 (1980) 3662.

[24] A. H. Fainberg, S. Winstein, J. Am. Chem. Soc. 78 (1956) 2770.

[25] H. Elias, G. Gumbel, S. Neitzel, H. Volz, Z. Anal. Chem. 306 (1981) 240

[26] H. Langhals, Nouv. J. Chim. 5 (1981) 97.

[27] E. Tommila, Acta Chem. Scand. 9 (1955) 975.

[28] H. Langhals, Z. Anal. Chem. 305 (1981) 26.

[29] H. Langhals, Chem. Ber. 114 (1981) 2907.

[30] H. Langhals, Z. Phys. Chem. (Frankfurt am Main) 127 (1981) 45.

[31] H. Langhals, Nouv. J. Chim. 5 (1981) 511.

[32] E. Grunwald, S. Winstein, J. Am. Chem. Soc. 70 (1948) 846

[33] S. Hünig, O. Rosenthal, Liebigs Ann. Chem. 592 (1955) 161.

[34] M. J. Minch, S. S. Shah, J. Chem. Educ. 54 (1976) 709.

[35] I. A. Zmyreva, V. V. Zelinsky, V. P. Kolobkov, N. D. Krasnickaja, Dokl. Akad. Nauk SSSR 129 (1959) 1089.

[36] V. V. Zelinski, V. P. Kolobkov, L. G. Pikulik, Opt. Spektrosk. I (1956) 161.

[37] T. V. Veselova, I. I. Reznikova, A. S. Cherkasov, V. I. Shirokov, Opt. Spectrosc. USSR 25 (1968) 50.

[38] M. Chastrette, Tetrahedron 35 (1979) 1441.

[39] M. J. Blandamer, J. Burgess, Chem. Soc. Rev. 4 (1975) 55.

[40] H. Langhals, Z. Anal. Chem. 308 (1981) 441.
[41] Z. B. Maksimovic, C. Reichardt, A Spiric, Z Anal. Chem. 270 (1974) 100

[42] J. A. Howard, K. U. Ingold, Can. J. Chem. 42 (1964) 1044

[43] E. Lippert, W. Lüder, F. Moll, W. Nägele, H. Boos, H. Prigge, I. Seibold-Blankenstein, Angew. Chem. 73 (1961) 695 und zit. Lit.

[44] H. Langhals, Nachr. Chem. Tech. Lab. 28 (1980) 716.

[45] N. G. Bakhshiev, Opt. Spectrosc. USSR 16 (1964) 446.

[46] H. Langhals, Nouv. J. Chim. 6 (1982) 285.

[47] P. Suppan, Nouv. J. Chim., 6 (1982) 285.

[48] E. M. Arnett, W. G. Bentrude, J. J. Burke, P. McC. Duggleby, J. Am. Chem. Soc. 87 (1965) 1541.

[49] E. M. Arnett, W. G. Bentrude, P. McC. Duggleby, J. Am. Chem. Soc. 87 (1965) 2048.

[50] E. M. Arnett, P. McC. Duggleby, J. J. Burke, J. Am. Chem. Soc. 85 (1963) 1350.

[51] M. H. Abraham, J. Chem. Soc. Perkin Trans. II 1977, 1028

[52] L. Menninga, J. B. F. N. Engberts, J. Org. Chem. 41 (1976) 3101.

[53] W. Good, D. B. Ingham, J. Stone, Tetrahedron 31 (1975) 257.

[54] L. P. Hammett: Physikalische Organische Chemie, Verlag Chemie, Weinheim 1973; Physical Organic Chemistry, McGraw-Hill, New York 1970.

[55] R. Huq, J. Chem. Soc. Faraday Trans. I 1973, 1195.

[56] H. A. J. Holtermann, J. B. F. N. Engberts, J. Phys. Chem. 83 (1979) 443.

[57] E. M. Diefallah, M. A. Mousa, M. A. Ashy, S. A. Ghonaim, Z. Phys. Chem. (Frankfurt am Main) 115 (1979) 89.

[58] J. Moravcova, M. Vecera, Collect. Czech. Chem. Commun. 44 (1979) 2639.

[59] J. F. J. Engbersen, J. B. F. N. Engberts, J. Am. Chem. Soc. 97 (1975) 1563.

60] D. N. Kevill, A. Wang, J. Chem. Soc. Chem. Commun. 1981, 83.

[61] J. Eibenberger, D. Schulte-Frohlinde, S. Steenken, J. Phys. Chem. 84 (1980) 704.

[62] J. S. Lomas, J. Org. Chem. 46 (1981) 412

[63] A. D. Allen, M. P. Jansen, K. M. Koshy, N. N. Mangru, T. T. Tidwell, J. Am. Chem. Soc. 104 (1982) 207.

[64] S. Sridharan, V. P. Vitullo, J. Am. Chem. Soc. 99 (1977) 8093.

[65] I. Mergelsberg, H. Langhals, C. Rüchardt, Chem. Ber. 113 (1980) 2424

[66] T. W. Bentley, C. T. Bowen, H. C. Brown, F. J. Chloupek, J. Org. Chem. 46 (1981) 38

[67] H. Langhals, Z. Anal. Chem. 310 (1982) 427.

[68] K. Dimroth, C. Reichhardt, Z. Anal. Chem. 215 (1966) 344.

[69] C. Rüchardt, Top. Curr. Chem. 6 (1966) 251.

[70] H. Langhals, Angew. Chem. 94 (1982) 452; Angew. Chem. Int. Ed. Engl. 21 (1982) 432; Angew. Chem. Suppl. 1982, 1138.

[71] H. Langhals, Nouv. J. Chim. 6 (1982) 265. 